\title{
Interactions of Whisking and Touch Signals in the Rat Brainstem
}

\author{
Coralie Ebert, ${ }^{1}$ Knarik Bagdasarian, ${ }^{1}$ Sebastian Haidarliu, ${ }^{1}$ Ehud Ahissar, ${ }^{1}$ and Avner Wallach ${ }^{2}$ \\ ${ }^{1}$ Weizmann Institute of Science, Rehovot, Israel 7610001, and ${ }^{2}$ Columbia University, New York, New York 10027
}

Perception is an active process, requiring the integration of both proprioceptive and exteroceptive information. In the rat's vibrissal system, a classical model for active sensing, the relative contribution of the two information streams was previously studied at the peripheral, thalamic, and cortical levels. Contributions of brainstem neurons were only indirectly inferred for some trigeminal nuclei according to their thalamic projections. The current work addressed this knowledge gap by performing the first comparative study of the encoding of proprioceptive whisking and exteroceptive touch signals in the oralis $(\mathrm{SpVo})$, interpolaris $(\mathrm{SpVi})$, and paratrigeminal $(\mathrm{Pa} 5)$ brainstem nuclei. We used artificial whisking in anesthetized male rats, which allows a systematic analysis of the relative contribution of the proprioceptive and exteroceptive information streams along the ascending pathways in the absence of motor or cognitive top-down modulations. We found that (1) neurons in the rostral and caudal parts of the SpVi convey whisking and touch information, respectively, as predicted by their thalamic projections; (2) neurons in the SpVo encode both whisking and (primarily) touch information; and (3) neurons of the Pa5 encode a complex combination of whisking and touch information. In particular, the Pa5 contains a relatively large fraction of neurons that are inhibited by active touch, a response observed so far only in the thalamus. Overall, our systematic characterization of afferent responses to active touch in the trigeminal brainstem approves the hypothesized functions of SpVi neurons and presents evidence that $\mathrm{SpVo}$ and $\mathrm{Pa} 5$ neurons are involved in the processing of active vibrissal touch.

Key words: active sensing; active touch; brainstem processing; parallel pathways; proprioception; vibrissae

Significance Statement

The present work constitutes the first comparative study of the encoding of proprioceptive (whisking) and exteroceptive (touch) information in the rat's brainstem trigeminal nuclei, the first stage of vibrissal processing in the CNS. It shows that (1) as expected, the rostral and caudal interpolaris neurons convey primarily whisking and touch information, respectively; (2) the oralis nucleus, whose function was previously unknown, encodes both whisking and (primarily) touch touch information; (3) a subtractive computation, reported at the thalamic level, already occurs at the brainstem level; and (4) a novel afferent pathway probably ascends via the paratrigeminal nucleus, encoding both proprioceptive and exteroceptive information.

\section{Introduction}

Rats typically explore their environment by moving the long vibrissae located on each side of their snout. Sensory information gathered through this whisking behavior is based on reafferent and exafferent signals, both encoded by mechanical deformations

\footnotetext{
Received June 2, 2020; revised Mar. 15, 2021; accepted Mar. 16, 2021.

Author contributions: C.E., K.B., E.A., and A.W. designed research; C.E., K.B., and S.H. performed research; C.E., K.B., and S.H. analyzed data; C.E. wrote the first draft of the paper; C.E., K.B., S.H., E.A., and A.W. wrote the final version of the paper.

This work was supported by European Research Council under the EU Horizon 2020 Research and Innovation Program Grant Agreement 786949, Minerva Foundation funded by the Federal German Ministry for Education and Research, United States-Israel Binational Science Foundation Grant 2017216, and Irving B. Harris Fund for New Directions in Brain Research. E.A. holds the Helen Diller Family Professorial Chair of Neurobiology. We thank Martin Deschênes and Ilan Lampl for helpful comments and discussions.

The authors declare no competing financial interests.

Correspondence should be addressed to Avner Wallach at aw3057@columbia.edu or Ehud Ahissar at ehud.ahissar@weizmann.ac.il.

https://doi.org/10.1523/JNEUROSCI.1410-20.2021

Copyright $\odot 2021$ the authors
}

within the whiskers' follicles (Ebara et al., 2002; Szwed et al., 2003; Simony et al., 2010; Haidarliu et al., 2011; Boubenec et al., 2012; Quist et al., 2014; Tonomura et al., 2015; Severson et al., 2017). This information is sent from the trigeminal ganglion (TG) to the brainstem trigeminal complex, where it is propagated via at least three parallel pathways (Diamond et al., 2008; Deschênes and Urbain, 2009). The lemniscal pathway, arising from the principalis nucleus (PrV) and projecting to the dorsomedial sector of the ventral posteromedial nucleus (VPMdm) (Williams et al., 1994; Henderson and Jacquin, 1995; CastroAlamancos, 2002; Deschênes et al., 2003), conveys a combination of whisking and touch information (C. Yu et al., 2006; Moore et al., 2015). The rostral part of the interpolaris nucleus (SpVir) belongs to the paralemniscal pathway, which conveys information about whisker motion (C. Yu et al., 2006) to the posterior medial nucleus (POm) of the thalamus (Veinante et al., 2000). The caudal part of the interpolaris (SpVic) projects to the ventrolateral sector of the VPM (VPMvl) (Veinante et 
al., 2000) and is part of the extralemniscal pathway, which encodes touch information at the thalamic level (C. Yu et al., 2006). The oralis nucleus ( $\mathrm{SpVo}$ ) projects to the most caudal parts of POm and VPM (Jacquin and Rhoades, 1990; Veinante et al., 2000). It has been proposed that SpVo belongs to a "fifth pathway" whose function is yet unknown (Deschênes and Urbain, 2009) and that it is involved in a touch reflex (Bellavance et al., 2017). Previous anatomic works, based on cytoarchitectural considerations, have suggested that SpVo is constituted of two distinct subnuclei along the rostro-caudal axis in the rabbit (Meessen and Olszewski, 1949) and in the rat (Shigenaga and Yoshida, 2007). It is however unclear whether this division between the rostral and caudal parts of $\mathrm{SpVo}$ is also functional.

Last, the paratrigeminal nucleus $(\mathrm{Pa} 5)$ is a small nucleus located dorsal to SpVir (Chan-Palay, 1978). Pa5 was previously shown to play a role in cardiovascular regulation (Couture and Lindsey, 2000; Buck et al., 2001; Y. G. Yu et al., 2002; Junior et al., 2004; Caous et al., 2008; Sousa and Lindsey, 2009) and in nociception (Bon et al., 1997; Ma et al., 2005; Koepp et al., 2006) but never as being part of a vibrissal sensory pathway. However, it receives sensory inputs from trigeminal primary afferents (Marfurt and Rajchert, 1991; Sugimoto et al., 1997) and projects to the VPM (Saxon and Hopkins, 1998; Caous et al., 2001), which accommodates both the lemniscal and extralemniscal pathways (Pierret et al., 2000; C. Yu et al., 2006). We therefore posit that $\mathrm{Pa} 5$ may be involved in the afferent processing of vibrissal sensory information.

Encoding of either whisking or touch information by these different brainstem trigeminal areas has only been inferred so far from their thalamic projections. Previous electrophysiological studies conducted in the brainstem trigeminal nuclei focused on the neuronal responses in anesthetized animals to passive stimuli (Jacquin et al., 1986; Gibson, 1987; Sosnik et al., 2001; Moreno et al., 2005; Furuta et al., 2006, 2008; Mohar et al., 2013, 2015) or only examined the neuronal response to free-air whisking (Moore et al., 2015; Wallach et al., 2016).

The aim of the present study was to compare the responses of cells located in the rostral and caudal parts of both SpVo and $\mathrm{SpVi}$ and in Pa5 to whisking in free air and against an object, thus providing an account of whisking and touch selectivity in the brainstem trigeminal complex, and to compare them with the ones observed in the TG and thalamus. For this, we used a similar methodology (i.e., eliciting artificial whisking in the presence and absence of an object in anesthetized animals) as the one used by Szwed et al. (2003) in the TG and by C. Yu et al. (2006) in the thalamus. Using this method in the anesthetized model enables accurate delivery of tightly controlled identical stimuli, both with and without object contacts, while maintaining the natural context of active vibrissal motion. This allows for systemic characterization of the sensory drive and its processing in these regions in the absence of top-down modulation from motor or cognitive sources, and complements studies in awake behaving animals, which are harder to control and where such modulations are present.

\section{Materials and Methods}

Surgical and electrophysiological recording procedures. Experimental procedures were approved by the Institutional Animal Care and Use Committee of the Weizmann Institute of Science. Electrophysiological recordings were carried out on 29 male Wistar rats $(330 \pm 35 \mathrm{~g})$. Surgical procedures and electrophysiological recordings were performed as previously described (Szwed et al., 2003; Derdikman et al., 2006; C.
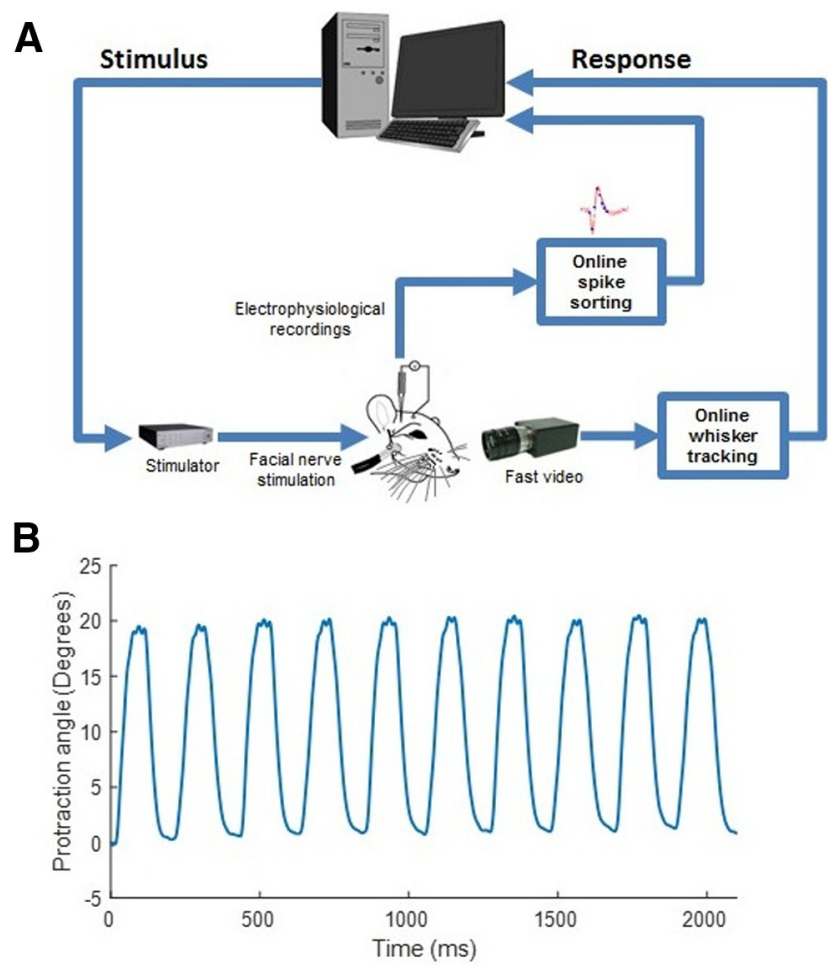

Figure 1. Experimental setup. $\boldsymbol{A}$, Experimental scheme. $\boldsymbol{B}$, Online tracking of a single whisker.

Yu et al., 2006; Wallach et al., 2016). Briefly, general anesthesia was induced using intraperitoneal injections of urethane $(1.5 \mathrm{~g} / \mathrm{kg}$ of body weight), reflexes were constantly monitored, and supplemental injections of urethane ( $10 \%$ of the initial dose) were given when required. Atropine methyl nitrate ( $0.3 \mathrm{mg}$ per $\mathrm{kg}$ of body weight) was administered intramuscularly to prevent respiratory complications, and the body temperature was maintained at $37^{\circ} \mathrm{C}$ using a regulated heating pad. Anesthetized animals were secured in a stereotaxic device (SR-6; Narishige). A craniotomy was made over the left trigeminal nucleus according to stereotaxic coordinates (Paxinos and Watson, 2006), and a tungsten microelectrode (0.8-1 M ; Alpha Omega Engineering) was lowered until single units drivable by manual whisker deflections were found. Single units were isolated using a template-based online spikesorter (ALAB-ASD3.1; Alpha-Omega Engineering). The receptive field and the principal whisker were determined for each recorded unit using manual whisker stimulation and auditory feedback. Active artificial whisking was evoked by electrical stimulation of the facial nerve. Briefly, the left buccal motor branch of the facial nerve was isolated at the middle cheek level of the face and mounted on a pair of custom-made silver hook electrodes (Szwed et al., 2003).

Experimental paradigms. Stimulations were given in blocks of 24 trains of artificial whisking, following a similar protocol as in Szwed et al. (2003), C. Yu et al. (2006), and Wallach et al. (2016) (Fig. 1A). Each train contained 10 whisking cycles $(5 \mathrm{~Hz}, 50 \%$ duty cycle) lasting $2 \mathrm{~s}$, followed by a resting period of $2 \mathrm{~s}$. Thus, each block lasted $24 \times 4=96$ s. Artificial whisking was achieved via biphasic, rectangular electrical pulses (0.8-1.5 $\mathrm{V}$ amplitude, $30 \mu$ s duration) that were delivered to the facial nerve via the hook electrodes at $50 \mathrm{~Hz}$, using a digital-toanalog converter (NI PCI-6221, National Instruments). For generating each single whisking cycle, the stimulus was given for $100 \mathrm{~ms}$ (5 biphasic pulses) to induce protraction, followed by $100 \mathrm{~ms}$ pause to allow whiskers retraction back to their initial position. All the whiskers were left intact, and one whisker was slightly stained using an eyeliner to facilitate online tracking. Whisker movements were recorded and tracked online at 500 frames/s with a fast-digital video camera (Basler 504k, Basler). An example of online tracking of artificial whisking is presented in Figure $1 B$. 
Video recordings were synchronized with neurophysiological data at $1 \mathrm{~ms}$ accuracy, and object-contact times were extracted offline from the video recordings. Sessions were performed alternatively for both free-air whisking and whisking against an object (a 2-mm-diameter vertical pole positioned at $70 \%-90 \%$ of the principal whisker's radial length and $1 \mathrm{~mm}$ rostrally from the whisker resting position). For most of the cells (93\%), the whole procedure was repeated 3 times, thus totaling a number of $720(3 \times 24 \times 10)$ whisking cycles per object condition.

Management of the experimental setup (extracellular and video recordings, whisker stimulation, and tracking) was performed using a custom-made real-time application (Labview, National Instruments) described by Wallach et al. (2016).

Histology. At the end of each experiment, electrolytic lesions were induced by passing current $(100 \mu \mathrm{A}$ for $3 \mathrm{~s}$, unipolar) through the tip of the recording electrode. The animals were killed using intraperitoneal penthal injections; and the brainstem was then removed, fixed in $4 \%$ PFA with $25 \%$ sucrose, sliced parasagittaly to $60 \mu \mathrm{m}$ slices, and stained for cytochrome oxidase (Haidarliu and Ahissar, 2001). After staining, the lesions could be clearly seen and the brainstem nuclei, from which the recordings were performed, could be easily identified (Fig. 2B).

Previous studies suggested that $\mathrm{SpVo}$ is constituted of two histologically distinct parts along the rostro-caudal axis (Meessen and Olszewski, 1949; Falls, 1984; Jacquin and Rhoades, 1990; Shigenaga and Yoshida, 2007). As we have also observed, this separation, the distinction between the rostral (SpVor) and caudal (SpVoc) parts of SpVo, was kept during the study and the cell responses in those areas were analyzed separately.

Analysis of neuronal data. Single-unit recordings were performed in SpVor ( 7 cells, 5 rats), SpVoc (32 cells, 5 rats), SpVir (32 cells, 6 rats), SpVic ( 28 cells, 6 rats), and Pa5 ( 33 cells, 7 rats). A schematic representation of the location of the recordings is presented in Figure $2 A$. For every cell, we compared the stimulus-evoked response to the spontaneous activity (50 ms moving window averaging). Only cells whose evoked firing rate, either in the "free-air whisking" or in the "whisking against an object" conditions, surpassed the mean spontaneous rate by 2 SDs were considered in the study.

Whisking onset time and contact time were determined manually from the video recordings as the times at which the whiskers started moving. Raster plots and peristimulus time histograms (PSTHs; $1 \mathrm{~ms}$ bins, smoothed by a moving average with a $5 \mathrm{~ms}$ window) were computed and examined for all trains of each cell. The times of the initial responses to whisking and to touch were defined as the first instances where the spike probabilities were significantly different from the spontaneous spike probability.

The touch index (TI) of the cells was defined as described by C. Yu et al. (2006) as $T I=\frac{S_{T}-S_{W}}{S_{T}+S_{W}}$, with $S_{\mathrm{T}}$ and $S_{\mathrm{W}}$ being, respectively, the spike count during whisking against an object and free-air whisking (summed within a $150 \mathrm{~ms}$ time window starting at protraction onset). Four cell types were defined based on the TI (C. Yu et al., 2006). Following C. Yu et al. (2006), recorded units were considered to be touch (T) cells if the $\mathrm{TI}>0.8$, whisking + touch $(\mathrm{W}+\mathrm{T})$ cells if the TI was between 0.2 and 0.8 , whisking $(\mathrm{W})$ cells if the TI was between -0.2 and 0.2 , and whisking - touch (W-T) cells if the TI was $<-0.2$. Touch cells were further divided into four subcategories according to the Szwed et al. (2003) definition: Contact cells were defined as cells that fired only for a brief period of time after the whisker contacted the object; pressure cells presented a sustained activity throughout the whisker's contact with the object; detach cells were only active when the whisker started to retract and detach from the object; and finally, contact/detach responded both to contact and to detachment.

Average response latencies were computed from PSTHs as the delay from certain events (protraction onset or contact) to half-peak response (Sosnik et al., 2001). The duration of the neuronal response was measured as the period in which the neuronal activity was $>10 \%$ of the peak value.

Phase tuning analysis. To compute the phase tuning of each cell, we first translated the PSTH into a phase-response histogram $h(\varphi)$ by normalizing the time axis to the total cycle duration. Then we summed the phase response histogram in the complex plane to produce the unit's phase vector: $p_{\varphi}=\Sigma_{\varphi i=\{-\pi, \pi\}} h\left(\varphi_{i}\right) e^{i \varphi i}$. Therefore, the preferred phase of the unit is the argument (angle) of the phase vector, $\varphi=\operatorname{Arg}\left(p_{\varphi}\right)$, while the phase selectivity is the length of the vector, $\mathrm{S}=\left|p_{\varphi}\right|$ (for examples, see Wallach et al., 2016, their Fig. S3d).

Statistical analysis. Since the data did not follow a normal distribution, statistical differences (either between recording locations or between the different cell types) were computed using the KruskalWallis test. If a statistical difference between the groups was found, then a follow-up pairwise multiple comparison was performed. The functions kruskalwallis and multcompare from MATLAB R2017b (The MathWorks) were used for this purpose. The nonparametric Wilcoxon signed rank test (signrank function of MATLAB) was performed when comparing two or fewer conditions. The Hartigan's dip test (Hartigan, 1985; Hartigan and Hartigan, 1985) was used to test for the unimodality of the distribution of the TIs for monowhisker and multiwhisker cells. For the study of the adaptation of the neuronal response over a train (10 consecutive whisking cycles), the normalization of the spike count was done relative to the spike count in the first cycle, according to the following formula from Derdikman et al. (2006): normalized spike count $=$ $\frac{S_{i}-S_{1}}{S_{i}+S_{1}}$, where $S_{i}$ was the spike count in the $\mathrm{i}^{\text {th }}$ cycle and $\mathrm{S}_{1}$ the spike count in the first cycle. Thus, the normalized spike count attained positive values between 0 and 1 for facilitation and negative values from 0 to -1 for depression. The times of the initial response to whisking and the time of the object-induced suppression for W-T cells were calculated 


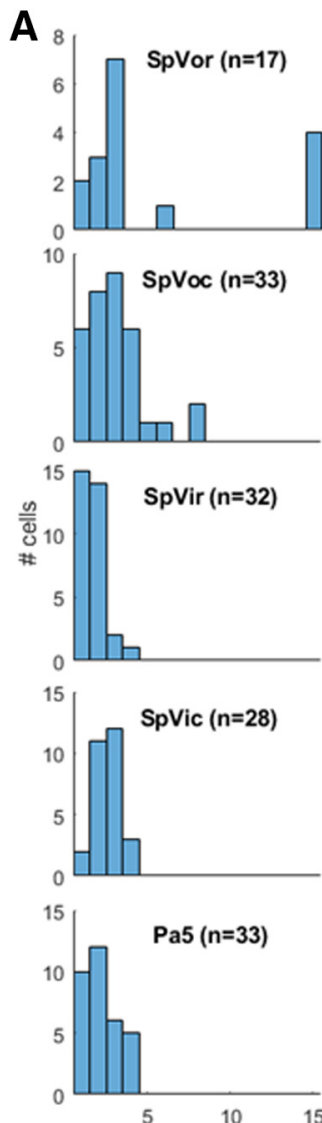

Size of the receptive field
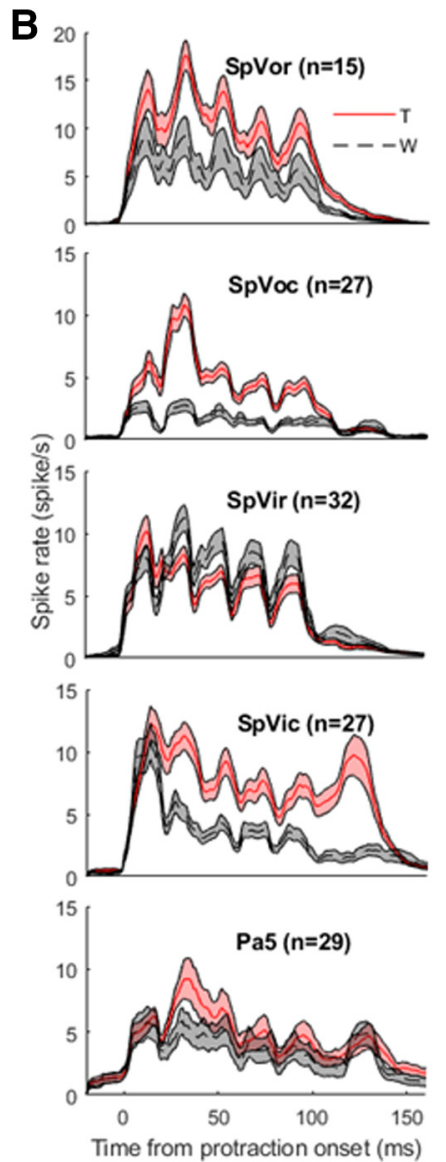
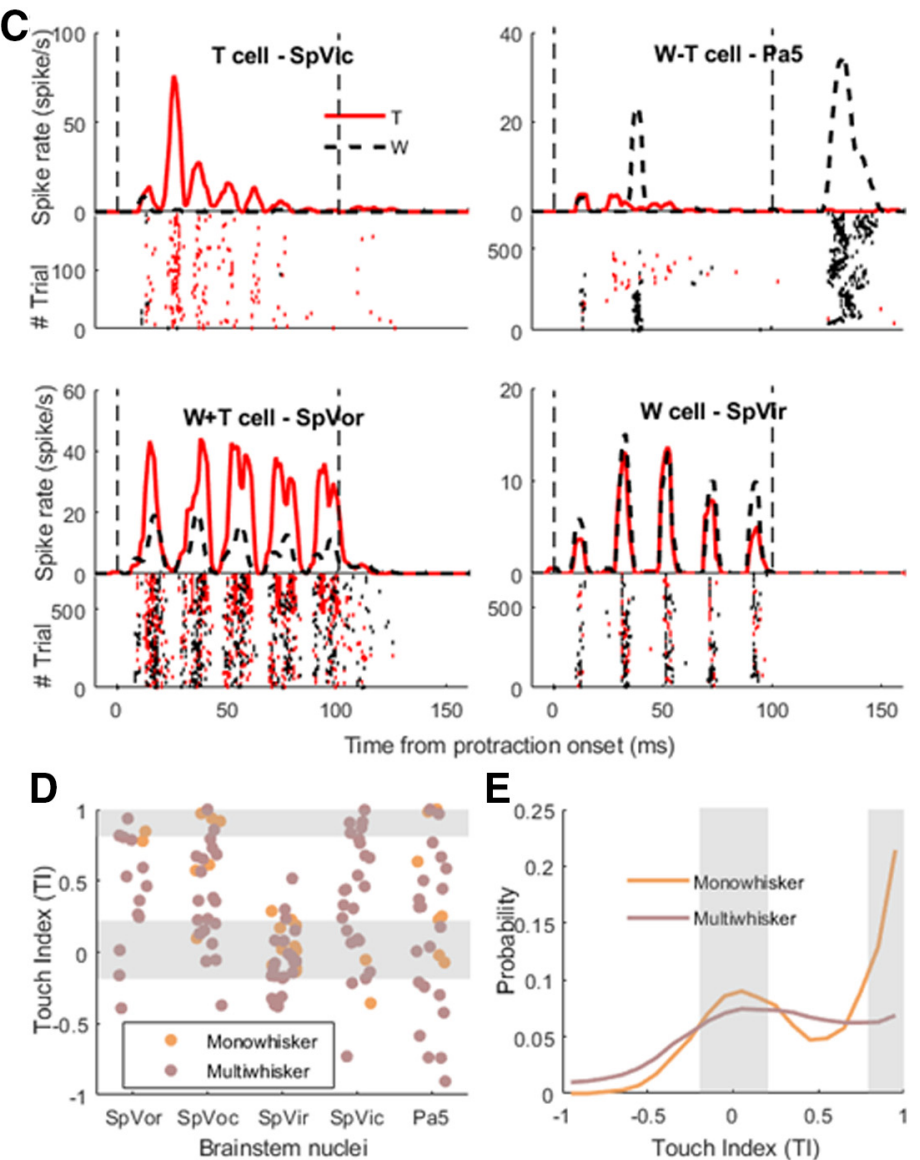

Figure 3. Receptive field and neuronal response of the cells depending on their brainstem location. $A$, Distribution of the size of the receptive field (number of whiskers to which the cell responds) for the five considered brainstem locations. $\boldsymbol{B}$, Population responses, averaged for each cell over all whisking cycles, to whisking in free-air (black) and against an object placed 1 mm rostral to the principal whisker (red). Shaded areas represent the SEM. The $50 \mathrm{~Hz}$ oscillations correspond to the electrical stimulus frequency. $\boldsymbol{C}$, Neuronal responses averaged over all whisking cycles of four single units to whisking in free-air (black) and against an object (red). Dashed lines indicate the onsets of protraction and retraction. $\boldsymbol{B}$, $\boldsymbol{C}$, The PSTHs and raster plots are aligned to whisker protraction onset. D, Tls of individual units $(T I=(S T-S W) /(S T+S W)$ with ST and SW corresponding to the spike counts for touch and whisking, respectively) for the different recorded locations (indicated on the $x$ axis). Orange dots represent monowhisker cells. Purple dots represent multiwhisker cells. $\boldsymbol{E}$, Distribution of the Tls for monowhisker (orange line) and for multiwhisker cells (purple line) for all the brainstem nuclei. The corresponding raw data are shown in $\boldsymbol{D}$.

with a $1 \mathrm{~ms}$ resolution by assuming a binomial distribution. Briefly, for each $1 \mathrm{~ms}$ time bin, we computed the $z$ score (the distance from the mean in units of SDs). For the binomial distribution, the $z$ score is defined as $Z=\frac{p_{1}-p_{2}}{\sqrt{p(1-p)\left(\frac{1}{n_{1}}+\frac{1}{n_{2}}\right)}}$, where $p_{1}$ and $p_{2}$ were the spike probability in each condition (conditions were as follows: free-air whisking, whisking against an object, spontaneous activity), $\mathrm{p}$ the total spike probability, and $n_{1}$ and $n_{2}$ the number of repeats for each condition. The spike probabilities for the two conditions were significantly different if $|Z| \geq 1.96$ (corresponding to the 95th quantile).

\section{Results}

In total, 142 cells were recorded and sorted online. All these cells responded to manual deflections of the whiskers and were considered for the receptive field analyses. Thirteen of these cells did not respond to free-air whisking or whisking against an object and were thus excluded from further quantitative analysis.

\section{The spatial extent of whisker receptive fields varies between nuclei}

We quantified and compared statistically the neurons' spatial receptive fields (number of whisker follicles driving each neuron) in the various nuclei. The average size of the whisker receptive field differed between the recording areas $\left(p=1.8 \times 10^{-5}\right.$; Kruskal-Wallis test; see Fig. $3 A$; Table 1). Units recorded in SpVir had on average a smaller receptive field than all other trigeminal brainstem nuclei $(p<0.005$; Kruskal-Wallis test, pairwise comparisons), at the exception of $\operatorname{Pa} 5(p=0.25)$. For the other nuclei, the differences in the size of receptive field were not statistically significant (pairwise comparisons based on Kruskal-Wallis test: $p>0.071)$. Yet, several cells in $\mathrm{SpVor}$ tended to have very large receptive fields, with some of them spanning up to three rows of whiskers (4 of 17 cells). Such cells were not observed in any of the other considered brainstem nuclei.

The proportion of monowhisker cells also varied between nuclei ( $p=0.0031$; Kruskal-Wallis test). The smallest proportion was recorded in SpVor and SpVic (2 of 17 and 28, respectively) and the highest proportion in SpVir (15 of 32) and Pa5 (10 of 33). Six monowhisker cells of 33 were recorded in SpVoc (18.2\%). The difference in the proportion of monowhisker cells between SpVir and the rest of the trigeminal nuclei was significant ( $p<0.05$; Kruskal-Wallis test, pairwise comparisons), but not when compared with $\mathrm{Pa} 5(p=0.22)$. In summary, whisker receptive fields were significantly smaller in SpVir and $\mathrm{Pa} 5$ and the cells presenting the largest receptive fields were found in SpVor. 
Table 1. Cell characteristics among brainstem nuclei ${ }^{a}$

\begin{tabular}{|c|c|c|c|c|c|c|}
\hline & SpVor & SpVoc & SpVir & SpVic & Pa5 & All cells \\
\hline Receptive field & $5.59 \pm 0.67$ & $3.03 \pm 0.15$ & $1.66 \pm 0.07$ & $2.5714 \pm 0.07$ & $2.18 \pm 0.09$ & $2.74 \pm 0.10$ \\
\hline Monowhisker cells & $2(11.76 \%)$ & $6(18.18 \%)$ & $15(46.88 \%)$ & $2(7.14 \%)$ & $10(30.30 \%)$ & $35(24.48 \%)$ \\
\hline $\mathrm{TI}$ & $0.46 \pm 0.05$ & $0.44 \pm 0.04$ & $-0.03 \pm 0.02$ & $0.33 \pm 0.04$ & $0.21 \pm 0.05$ & $0.25 \pm 0.02$ \\
\hline $\mathrm{T}$ cells $(\mathrm{TI}>0.8)$ & $4(26.67 \%)$ & $5(18.52 \%)$ & 0 & $5(18.52 \%)$ & $5(17.24 \%)$ & $19(14.62 \%)$ \\
\hline $\mathrm{W}+\mathrm{T}$ cells $(0.2<\mathrm{TI}<0.8)$ & $8(53.33 \%)$ & $14(51.85 \%)$ & $5(15.63 \%)$ & $11(40.74 \%)$ & $11(37.93 \%)$ & $49(37.69 \%)$ \\
\hline $\mathrm{W}$ cells $(-0.2<\mathrm{TI}<0.2)$ & $2(13.33 \%)$ & $7(25.93 \%)$ & $22(68.75 \%)$ & $8(29.63 \%)$ & $5(17.24 \%)$ & $44(33.85 \%)$ \\
\hline W-T cells $(\mathrm{TI}<-0.2)$ & $1(6.67 \%)$ & $1(3.70 \%)$ & $5(15.63 \%)$ & $3(11.11 \%)$ & $8(27.59 \%)$ & $18(13.85 \%)$ \\
\hline W-T cells $(\mathrm{TI}<-0.5)$ & 0 & 0 & 0 & $1(3.70 \%)$ & $4(13.79 \%)$ & $5(3.85 \%)$ \\
\hline Response latency (ms) & $7.26 \pm 0.42$ & $8.81 \pm 0.59$ & $9.39 \pm 0.34$ & $8.51 \pm 0.27$ & $9.61 \pm 0.54$ & $8.85 \pm 0.19$ \\
\hline Response duration (ms) & $98.73 \pm 2.96$ & $86.5 \pm 3.19$ & $90.31 \pm 2.81$ & $118.07 \pm 2.49$ & $105.69 \pm 3.76$ & $99.71 \pm 1.49$ \\
\hline
\end{tabular}

${ }^{a}$ The average size of the receptive field (number of whiskers to which each cell responded), the number of monowhisker cells, the average TI, the number of each cell type (computed based on the TI) as well as the average response latency (delay to half-peak from contact onset for touch cells or from protraction onset for the rest), and the average response duration (time during which the neuronal activity was $>10 \%$ of the maximal response) are indicated for each considered brainstem nucleus. When appropriate, percentages are also indicated in parentheses. All of the ranges correspond to the SEM.

\section{SpVor, SpVoc, and SpVic process primarily touch signals, whereas SpVir processes primarily whisking information} We analyzed the neuronal responses of the considered trigeminal nuclei to free-air whisking and whisking against an object. Our hypothesis was that SpVir and SpVic would encode whisking and touch information, respectively, based on their thalamic projections. We also hypothesized that the SpVor and SpVoc should encode mostly touch information, given their proposed role in a touch-induced reflexive loop (Bellavance et al., 2017). As predicted, cells located in SpVor, SpVoc, and SpVic had stronger responses to touch than to free-air whisking, whereas cells recorded in SpVir showed a similar response whether or not an object was present in their receptive field (Fig. $3 B$ ).

The relative selectivity for whisking and touch was calculated for each cell using the TI (see Materials and Methods). Cells were classified in four categories of active-sensing responses, based on their PSTHs and TIs (C. Yu et al., 2006, 2015): W, T, $\mathrm{W}+\mathrm{T}$, and $\mathrm{W}-\mathrm{T}$ (see Materials and Methods). An example cell for each category is presented in Figure $3 C$. Figure $3 D$ shows that SpVor, SpVoc, and SpVic contain $\mathrm{W}, \mathrm{W}+\mathrm{T}$, and $\mathrm{T}$ cells, whereas SpVir contains primarily whisking cells.

Szwed et al. (2003) further divided touch cells into four categories: contact cells, which fired for a short period of time after the whisker contacted the object; pressure cells, whose activity was sustained for as long as the whisker was pressing against the object; detach cells, which fired only when the whisker started to retract and detach from the object; and contact/detach cells, which responded to both events. Because of the small number of touch cells in each nucleus, this analysis was performed for all the brainstem nuclei together. The distribution between the four categories was not statistically different between the brainstem and the TG (Kruskal-Wallis test: $p=0.91$ ). Both brain areas presented a high proportion of pressure cells (37\% for the TG and $61 \%$ for the brainstem), and a reasonably high proportion of cells that responded during the retraction epoch (TG: $20 \%$ of detach cells and $16 \%$ of contact/detach cells; brainstem: $7 \%$ of detach cells and $18 \%$ of contact/detach cells); $27 \%$ and $14 \%$ of contact cells were found in the TG and the brainstem, respectively.

Monowhisker and multiwhisker cells presented different distribution of the TIs (Fig. 3E). Most monowhisker cells appeared to be either whisking or touch cells, which resulted in a bimodal distribution of the TIs ( $p=0.04$; Hartigan's dip test). This was not the case for multiwhisker units ( $p=0.18$; Hartigan's dip test). $\mathrm{W}$-T cells tended to be exclusively multiwhisker cells, with no monowhisker unit with a TI $<-0.5$ having been identified.

\section{Brainstem nuclei exhibited fast, persistent responses to artificial whisking cycles}

For each cell, we computed the latency and duration of the neuronal response to free air whisking and/or whisking against an object. Most cells presented response latencies (measured as the latency to the half-peak from either contact onset for touch cells or protraction onset for the rest of the cells, see Materials and Methods) between 5 and $8 \mathrm{~ms}$ (Fig. 4A), with an average response time of $\sim 12 \mathrm{~ms}$. These delays did not differ significantly between nuclei ( $p=0.38$; Kruskal-Wallis test; Fig. $4 B$ ) and across response types ( $p=0.48$; Kruskal-Wallis test; Fig. $4 C$ ). In addition, no difference in the latency was found between monowhisker and multiwhisker cells ( $p=0.39$; Kruskal-Wallis test).

Neuronal activity was generally sustained for the entire duration $(100 \mathrm{~ms})$ of the whisking cycle (Fig. $4 B$; also see PSTH in Fig. $3 B$ ) in all trigeminal nuclei, with only few outliers (8 cells of 101) presenting a response duration $<50 \mathrm{~ms}$. Units recorded from $\mathrm{Pa} 5$ showed a broader distribution, although the average duration was also $\sim 100 \mathrm{~ms}(105 \pm 20 \mathrm{~ms})$. Cells from SpVic appeared to demonstrate a slightly longer neuronal response $(118 \pm 12 \mathrm{~ms}$ ) that persisted into the whisker retraction (see below). This duration of the neuronal response was significantly longer than the one observed in SpVir ( $p=0.003$; Kruskal-Wallis test, pairwise comparison) and in SpVco ( $p=0.004$; KruskalWallis test, pairwise comparison). Thus, artificial whisking evoked sensory responses in the trigeminal and paratrigeminal brainstem with latencies mostly between 5 and $8 \mathrm{~ms}$ and durations typically covering the entire whisking cycle.

\section{A subset of cells responded to whisker retraction}

Whisking cycle phase has been shown to be a key variable governing neural coding in the vibrissal system (Kleinfeld and Deschênes, 2011). We therefore focused next on analyzing the phase preference across the studied neuronal populations. For this, the phase tuning of each cell (see Materials and Methods) was plotted in polar coordinates (Fig. 5A,B). Consistent with earlier studies using our method, the large majority of the cells responded preferentially during whisker protraction; however, several cells, located mainly in $\mathrm{SpVic}$, responded preferentially during the retraction phase when whisking against an object (Fig. 5A). Some cells also responded preferentially to free-air whisker retraction, most of those cells appearing to be $\mathrm{W}$-T cells.

In order to further analyze these findings, a statistical comparison of the response peak amplitudes during protraction and retraction was performed. This was done in the presence of an object and analyzed according to region (Fig. $5 C$ ), and in free-air 

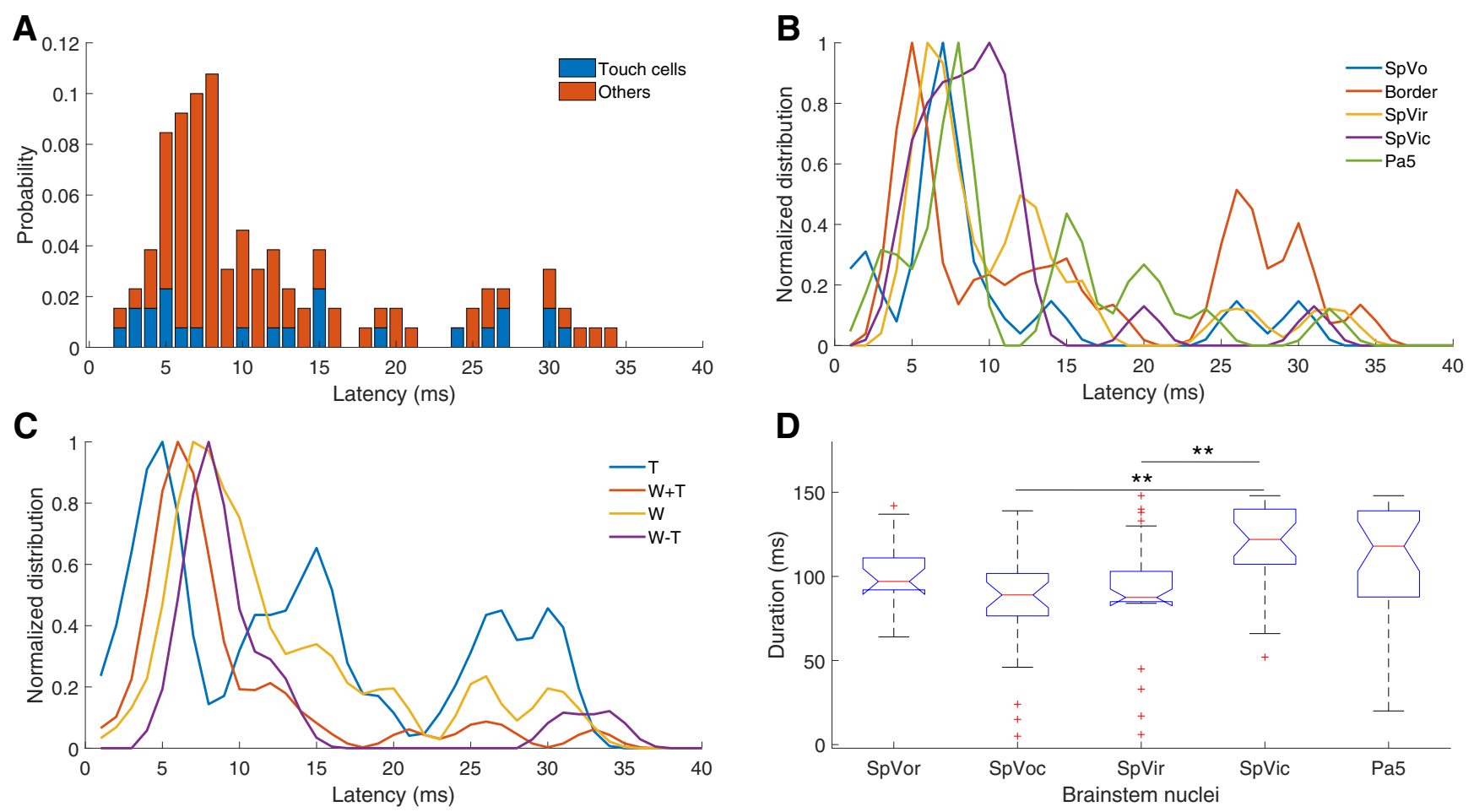

Figure 4. Latency and duration of the neuronal response to whisking against an object. $A$, Distribution of the latencies to the half-peak computed from touch onset (for touch cells; blue) or from protraction onset for the rest of the cells (red). $B, C$, Distribution of the latencies to the half-peak from touch onset (for touch cells) or from protraction onset (for the rest) for different recording locations $(\boldsymbol{B})$ or for different cell types (C). Histograms were smoothed using a Gaussian kernel (5 ms width). $\boldsymbol{D}$, Duration of the neuronal response computed as the time in which the neuronal activity was $>10 \%$ of the peak response. ${ }^{* *} p<0.01$ (Kruskal-Wallis test).

whisking and analyzed according to response type (Fig. 5D). Figure $5 E, F$ presents the normalized difference between the magnitude of the response to retraction and the maximum peak height during the protraction phase. Retraction responses were rarely observed in SpVir but were significantly more common in SpVic ( $p=0.0007$; Kruskal-Wallis test, pairwise comparison) and $\operatorname{Pa} 5$ ( $p=0.0009$; Kruskal-Wallis test, pairwise comparison) (Fig. 3B). W-T cells tended to show a more pronounced late peak component in free-air whisking ( $p=0.02$; Kruskal-Wallis test, pairwise comparison; Figs. 5 and $3 C$ ). Thus, brainstem retraction responses were more common in SpVic and $\mathrm{Pa} 5$ in the presence of an object and were mostly exhibited by W-T cells in free-air whisking.

\section{Brainstem and thalamus demonstrate similar neuronal selectivity in the paralemniscal pathway but not in the extralemniscal pathway}

Our results so far indicate that the whisking/touch selectivities observed in the paralemniscal and extralemniscal pathways in the thalamus are already present at the brainstem level. In order to quantify this similarity, the distribution of the TIs in the considered brainstem nuclei was compared with the ones measured in the TG (unpublished data from Szwed et al., 2003) and in the thalamus (C. Yu et al., 2006).

The distribution of TIs in TG covers a wide range with two salient peaks: one around $\mathrm{TI}=0$ (W cells) and one around $\mathrm{TI}=1$ ( $\mathrm{T}$ cells). In contrast, both the brainstem (SpVir) and thalamic (POm) stations of the paralemniscal pathway contained only neurons with TI $\sim 0$ (W cells; Fig. $6 A$ ). The difference in the distribution between TG and the two paralemniscal stations (SpVir and POm) is statistically significant ( $p<0.0033$; Kruskal-Wallis test, pairwise comparison), whereas the TI distributions of SpVir and POm were not significantly different from one another ( $p=0.87$; Kruskal-Wallis test, pairwise comparison).

In contrast to the preservation of selectivity from brainstem to thalamus along the paralemniscal pathway, the selectivity along the extralemniscal pathway appeared to develop along the brainstem-thalamus axis (Fig. 6A). Thus, stations in the brainstem showed less selectivity than the thalamic stations: neurons in SpVic exhibited similar TIs to those observed in the TG ( $p=0.9$; Kruskal-Wallis test) but smaller than those observed in VPMvl ( $p=0.03$; Kruskal-Wallis test); SpVor TIs exhibited a similar trend as those of SpVic, and SpVoc TIs showed a distribution that was in between those of the TG and VPMvl (Fig. $6 A)$. However, these differences were not statistically significant ( $p>0.17$; Kruskal-Wallis test, pairwise comparison).

\section{Longer response latencies in the paralemniscal compared with the extralemniscal pathways originate at the thalamic level}

As mentioned above, the response latency (measured as the delay to the half-peak) was similar across all considered brainstem nuclei. Next, we examined whether this observation is true at the thalamic level. The response latency at the TG, brainstem, and thalamic levels for the paralemniscal and extralemniscal pathways are presented in Figure 6B. As expected, the latency increases as the sensory signal propagates along the pathways. The average response latency is $3.95 \mathrm{~ms}$ in the TG, $12.37 \mathrm{~ms}$ in the brainstem, and $13.76 \mathrm{~ms}$ in the thalamus. However, while the response latency was comparable between the paralemniscal and extralemniscal pathway at the brainstem level, this does not seem to be the case at the thalamic level. In the extralemnical pathway, the thalamic response latency was only slightly higher than in the brainstem (12.23 ms vs $10.36 \mathrm{~ms})$, and the difference was not 
A Whisking against an object
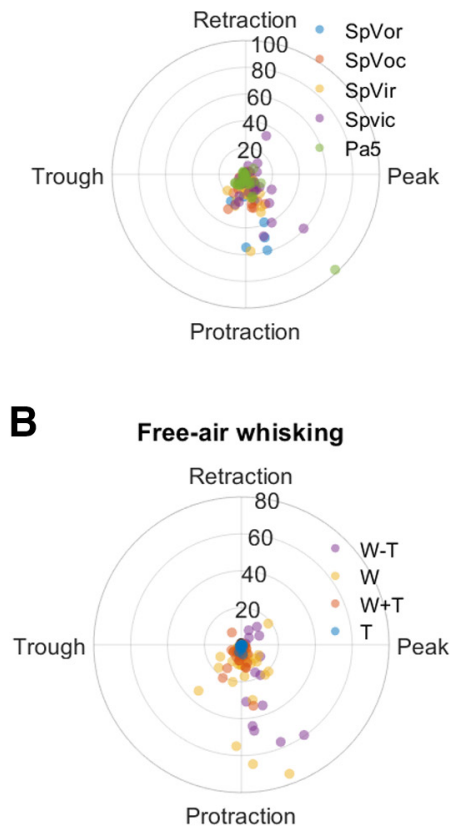

C
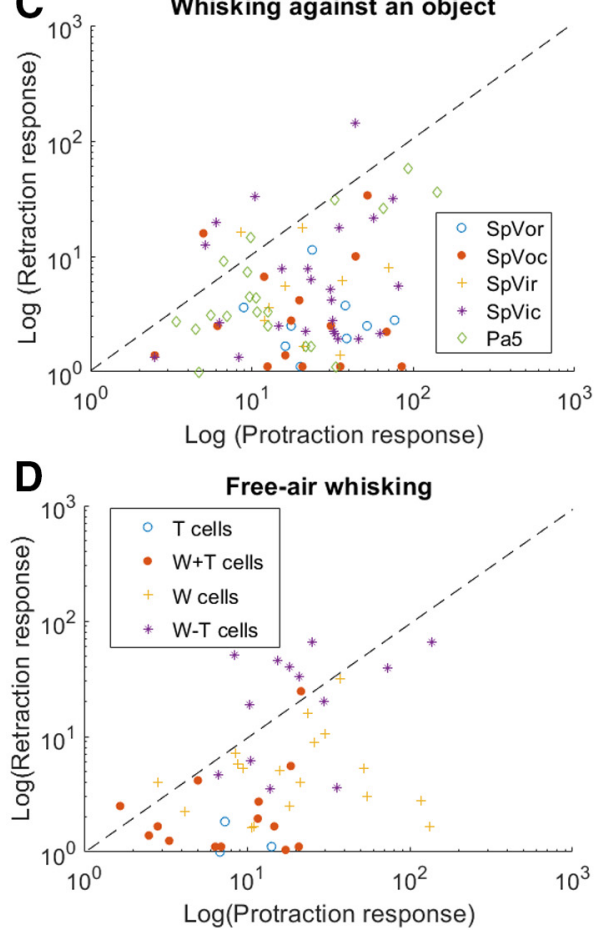

E

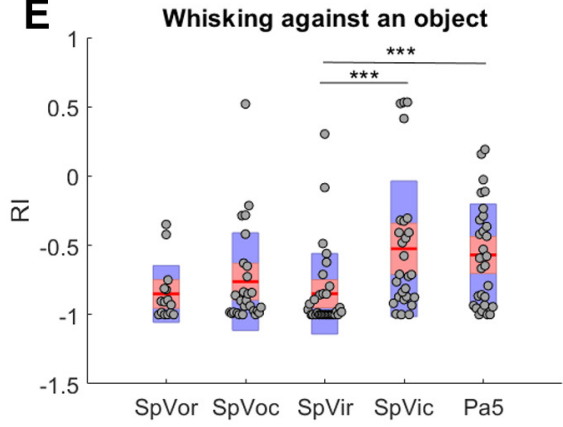

$\mathbf{F}$

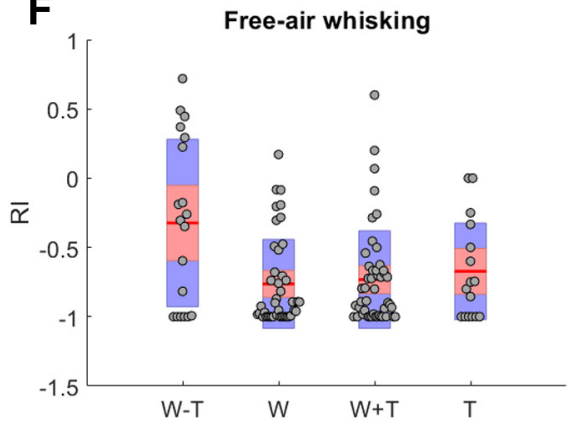

Figure 5. Response to whisker retraction. $\boldsymbol{A}, \boldsymbol{B}$, Polar plots showing the preferred phase of the cells (defined as the phase of the peak of the PSTH) and its magnitude (radial distance from the origin, in spikes/s) for whisking against an object $(\boldsymbol{A})$ and free-air whisking $(\boldsymbol{B})$. In both conditions, most cells appeared to respond during the protraction phase (bottom half of the graphs). C, $\boldsymbol{D}$, The two plots compare, for every recorded unit which presented a significant response to retraction, the respective height of the peak of the PSTH during whisker protraction ( $x$ axis) and during whisker retraction (y axis). C, Data are segregated according to the brainstem nuclei where the recordings were performed, while the segregation was done in graph $\boldsymbol{D}$ according to the cell type (W-T, W, W $+\mathrm{T}, \mathrm{T})$. Plot $\boldsymbol{C}$ corresponds to the responses during whisking against an object, whereas plot $\boldsymbol{D}$ indicates the responses to free-air whisking. $\boldsymbol{E}, \boldsymbol{F}$, Graphs represent the distributions of the retraction index (RI), which corresponds to the normalized difference between the response to whisker retraction (0-50 ms after retraction onset) and the response to whisker protraction (0-100 ms after protraction onset). The response levels were computed based on the peaks of the PSTHs for whisking against an object among different brainstem nuclei $(\boldsymbol{E})$ and for free-air whisking among different cell types $(\boldsymbol{F})$. Middle marks represent the average. Edges of the red boxes represent SEM. Edges of the blue box represent 1 SD. Gray dot represents a different unit. ${ }^{* *} p<0.001$ (Kruskal-Wallis test).

significant (Kruskal-Wallis test; $p=0.4352$ ), although this could result from a lack of power of the statistical test. However, in the paralemniscal pathway, the latency was significantly higher at the thalamus compared with the brainstem (17.09 ms vs $12.22 \mathrm{~ms}$; $p=0.0221$; Kruskal-Wallis test). We conclude that response latency is higher in the paralemniscal pathway compared with the extralemniscal pathway, and that this difference originates at the thalamus.

Spatial selectivity is conserved in the extralemniscal pathway but not in the paralemniscal pathway

As shown above, cells in SpVir presented on average a smaller receptive field than units recorded in other brainstem trigeminal nuclei. As the paralemniscal pathway encodes whisking information, we hypothesized that a higher spatial integration could be observed at the thalamic level, resulting in the opposite phenomena. We therefore compared the size of the receptive fields for cells recorded in POm and in VPMvl (Fig. 6C). Indeed, larger receptive fields were observed in $\mathrm{POm}$, with cells responding on average to 4 whiskers compared with only 1.9 whiskers in VPMvl. This difference was significant (Kruskal-Wallis test; $p<$ 0.001 ) and resulted from a decrease in the spatial selectivity from SpVir to POm (Kruskal-Wallis test; $p<0.001$ ), whereas the size of the receptive fields did not differ in the extralemniscal pathway between SpVic and VPMvl (Kruskal-Wallis test; $p=$ $0.2137)$. We conclude that the spatial selectivity is conserved between the brainstem and thalamic stations in the extralemniscal pathway, but not in the paralemniscal pathway, where the cells responded on average to more whiskers in the thalamus than in the brainstem.

\section{Pa5 neurons exhibit whisking responses that were suppressed by touch}

$\mathrm{Pa} 5$ was the only brainstem nucleus, in our sample, whose cells covered the entire range of the TI spectrum (from -1 to 1 ). $\mathrm{Pa} 5$ also accommodates the highest proportion of $\mathrm{W}$-T cells in our study (27.6\%; see example of a W-T cell in Fig. 3C).

We compared the Pa5's touch selectivity with the observations from VPMdm (Fig. 6A), a thalamic lemniscal nucleus also containing W-T cells (C. Yu et al., 2006), whose primary brainstem input arrives from $\mathrm{PrV} . \mathrm{Pa} 5$ indeed exhibits the wide range of TIs reported in VPMdm, but also a high proportion of T cells (TI close to 1) typical of VPMvl. Interestingly, the Pa5 had twice as many cells with a high negative TI (TI $<-0.5)(13.8 \%)$ than VPMdm (6.6\%). We conclude that both the anatomic projections of $\mathrm{Pa} 5$ and the whisking and touch responses of its neurons are consistent with it contributing to vibrissal processing in VPM.

\section{Adaptation to whisking and touch increases the cell} selectivity for $\mathrm{T}, \mathrm{W}+\mathrm{T}$, and $\mathrm{W}$ - $\mathrm{T}$ cells

As mentioned above, W-T cells, which are absent in the TG, were recorded in the brainstem. One possible mechanism for the generation of this response type is adaptation. In order to test this hypothesis, we quantified the dynamics of the response magnitude to protraction. To do so, we measured the spike count 

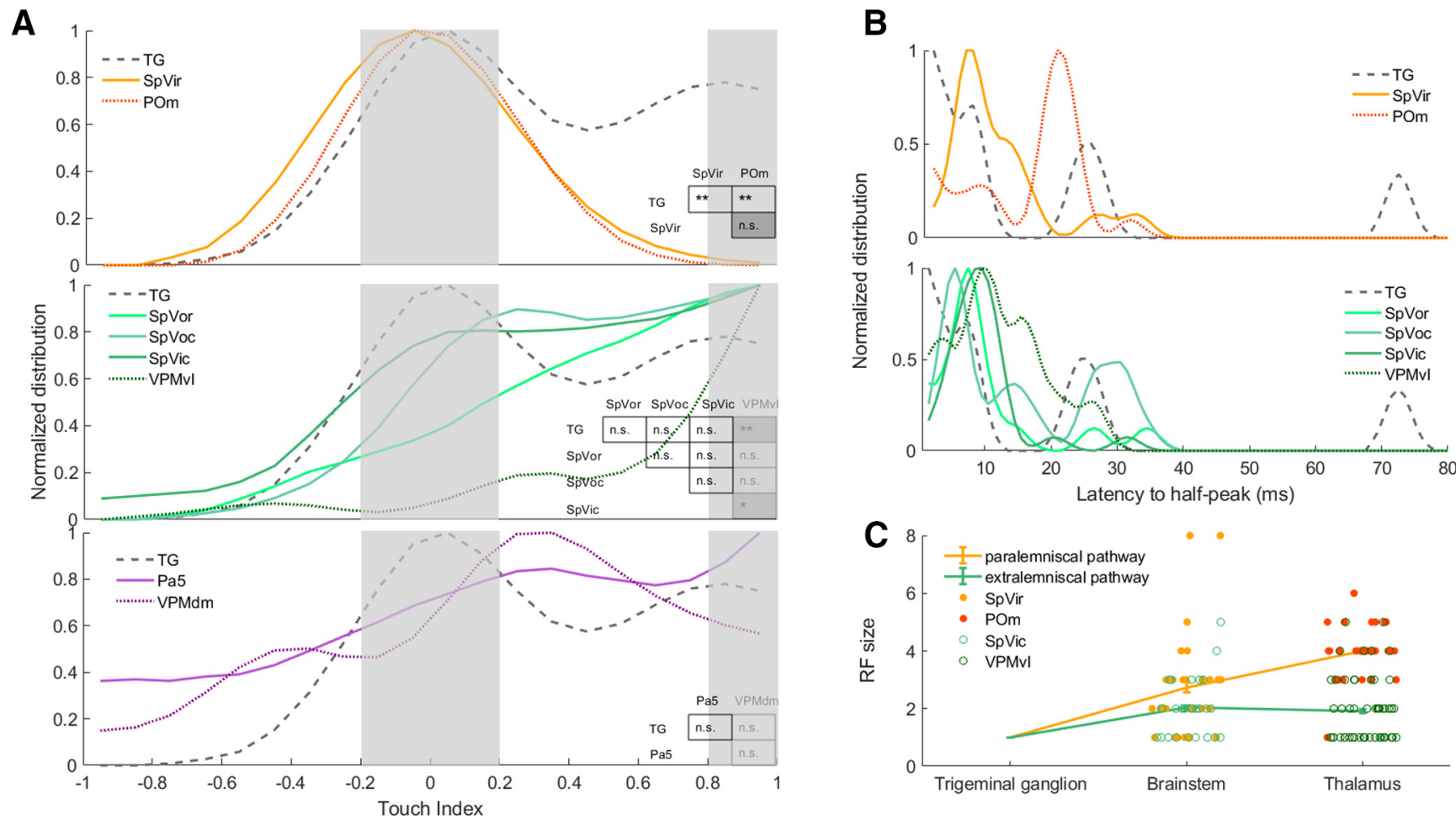

Figure 6. Comparison of the neuronal responses to whisking and touch stimuli between the TG, brainstem, and thalamus. $A$, Conservation of the touch selectivity along the afferent pathways. Top, Comparison of the distribution of the Tls in the paralemniscal pathway, from the TG to the posterior medial thalamic nucleus (POm) via the rostral interpolaris nucleus (SpVir). Middle, Data for region carrying mostly touch information (SpVor, rostral oralis nucleis; SpVoc, caudal oralis nucleus; SpVic, caudal interpolaris nucleus, in the brainstem and VPMvl, ventrolateral part of the ventro-posteromedial nucleus, in the thalamus). Bottom, Comparison of TIs distribution for areas presenting a high proportion of W-T cells (Pa5 in the brainstem and VPMdm in the thalamus). $\boldsymbol{B}$, Comparison of the latency distribution (computed as the delay to half-peak from contact onset for touch cells and from protraction onset for the rest of the cells) in the paralemniscal pathway (top plot, from the TG to the posterior medial nucleus POm via the rostral interpolaris nucleus SpVir) and in the extralemniscal pathway (bottom plot, from the TG to the ventrolateral part of the ventro-posteromedial nucleus VPMdm via the caudal interpolaris nucleus SpVic). The rostral and caudal part of the oralis nucleus (SpVor and SpVoc), which also show a preferred response to touch, were added to the later graph. C, Conservation of the spatial selectivity (indicated by the size of the receptive field) along the paralemniscal (orange; from the TG to the posterior medial nucleus POm via the rostral interpolaris nucleus SpVir) and extralemniscal (green; from the TG to the ventrolateral part of the ventro-posteromedial nucleus VPMdm via the caudal interpolaris nucleus SpVic) pathways. Error bars indicate SEs.

during the first $100 \mathrm{~ms}$ in each whisking cycle of a train (each train contained 10 successive whisking cycles) and compared it with the spike count during the first $100 \mathrm{~ms}$ in the first whisking cycle. Response adaptation differed depending on the cell's response type (Fig. $7 A$ ). T and W-T cells showed a significant level of adaptation to both whisking and touch stimuli $(p<0.003$; Wilcoxon test, comparison of the responses between the first and last cycle of a train). For $\mathrm{W}+\mathrm{T}$ cells, the adaptation to the whisking stimuli was only significant in free-air $\left(p=8.9 \times 10^{-5}\right.$ for freeair whisking vs $p=0.3$ for whisking against an object; Wilcoxon test). W cells' adaptation was not statistically significant ( $p=0.2$ for free-air whisking and $p=0.3$ for whisking against an object; Wilcoxon test). Adaptation behavior did not differ significantly between monowhisker and multiwhisker cells $(p=0.6$ for free-air whisking and $p=0.4$ for whisking against an object; Wilcoxon test).

Next, we quantified the change in the TI between the first and last whisking cycles of the train (Fig. $7 B, C)$. W-T cells exhibited a decrease $(p=0.006$ : Wilcoxon test $)$ and $\mathrm{W}+\mathrm{T}$ and T cells an increase $(p<0.001)$ in the TI along the train. No significant change in the TI was found between the first and last cycles for whisking cells ( $p=0.92$; Wilcoxon test). We conclude that $\mathrm{T}, \mathrm{W}+\mathrm{T}$, and $\mathrm{W}$ - $\mathrm{T}$ cells exhibited response adaptation that led to a higher selectivity for their preferred stimulus.
Delay of the object-induced suppression in W-T cells

Since the object-induced suppression observed in W-T cells was evident already during the first whisking cycle of each train, we investigated the possible mechanisms underlying this response type by looking at the response dynamics within a cycle. For each $\mathrm{W}$-T unit, we computed the time at which the neuronal activity significantly exceeded the baseline activity (i.e., the initial response latency), for both free-air whisking and whisking against object. Then, we computed the time at which the neuronal response when whisking against an object became significantly smaller than in free-air (i.e., the latency of the touch-induced suppression). This was measured for all W-T units recorded in the trigeminal (SpVir, SpVic, SpVor, SpVoc) and paratrigeminal $(\mathrm{Pa} 5)$ nuclei, as well as for $\mathrm{W}$ - $\mathrm{T}$ thalamic cells (reuse of data published in C. Yu et al., 2006). These latencies were determined by standardizing the data ( $z$ score; see Fig. $8 A$ ) and comparing with a random null-hypothesis (see Materials and Methods).

$\mathrm{W}$-T cells recorded in the trigeminal nuclei, in the $\mathrm{Pa} 5$, and in the thalamus presented an initial response to whisking whose timing did not depend on the presence of an object (Fig. $8 B$ ) and whose amplitude was not significantly affected by the introduction of an object in the whisking field ( $p=0.43$; Wilcoxon test). As illustrated in Figure $8 C$, this initial response happened on average later for $\mathrm{Pa} 5$ neurons $(9.6 \pm 0.1 \mathrm{~ms})$ than for trigeminal neurons $(8.7 \pm 1.4 \mathrm{~ms})$ and, as expected, later for thalamic cells 

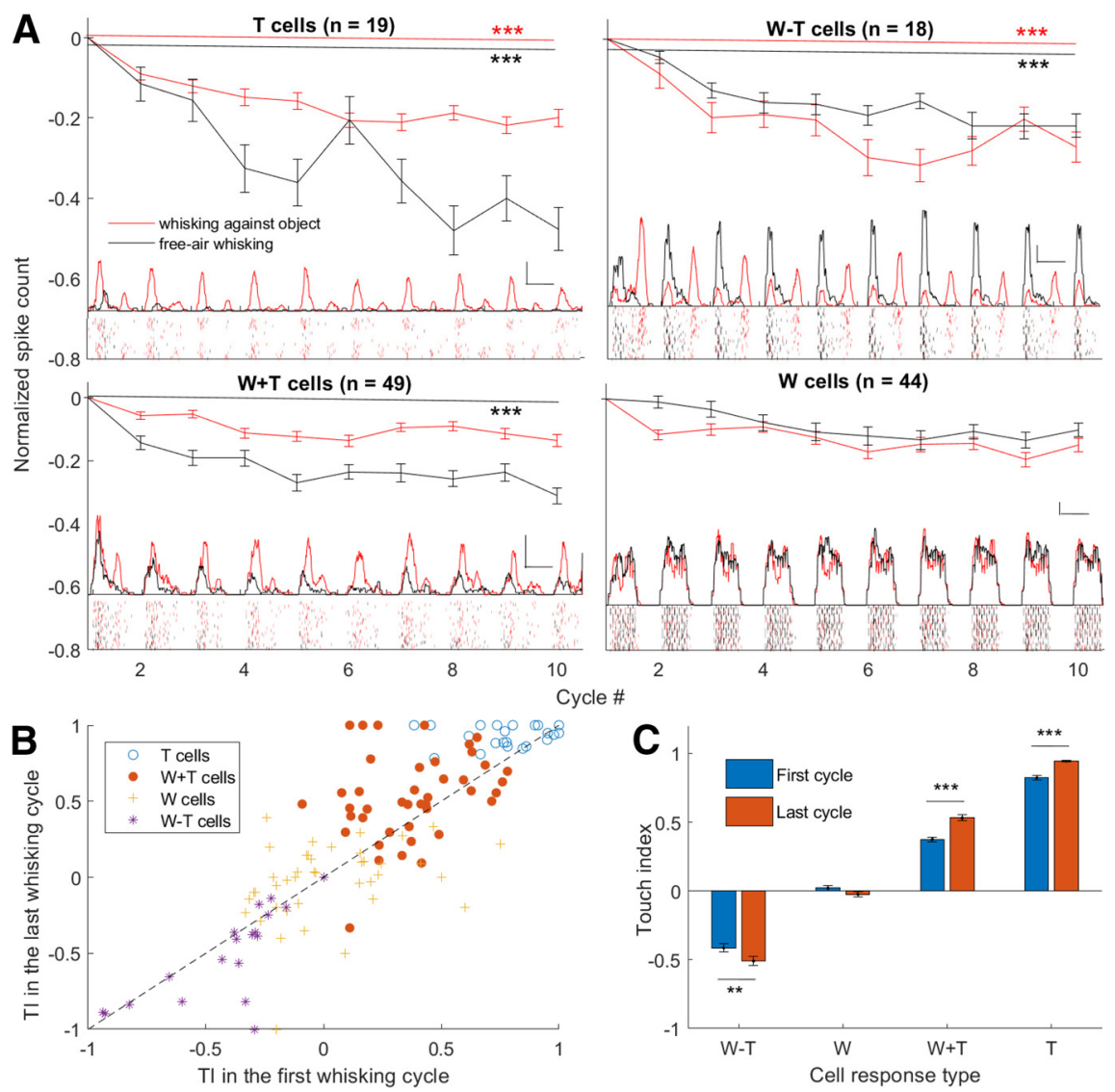

Figure 7. Strength of adaptation is inversely related to the cell preference. $\boldsymbol{A}$, Average normalized spike count during artificial whisking in free-air (black) and against an object (red). Spike counts were measured during the first $100 \mathrm{~ms}$ from protraction onset and normalized according to the response in the first cycle. Error bars indicate SEM. Small inserts on the bottom of each graph correspond to examples of the PSTH and raster plot of a single unit from the corresponding cell type computed over the 10 whisking cycles of a train. The scale of the PSTH is indicated by the $L$ on the right side. Vertical bar, 10 spikes/s; horizontal bar, $100 \mathrm{~ms}$. B, Comparison between the TI during the first ( $x$ axis) and the last ( $y$ axis) whisking cycle of a train, depending on the cell type. Each point corresponds to a different unit. C, Average TI (TI $=(S T-S W) /(S T+S W)$ with ST and SW corresponding to the spike counts for touch and whisking, respectively) for $\mathrm{W}-\mathrm{T}, \mathrm{W}, \mathrm{W}+\mathrm{T}$, and $\mathrm{T}$ cells for the first (blue) and the last (red) whisking cycles in a train (10 repetitions). Error bars indicate SEM. ${ }^{* *} p<0.01$; ${ }^{* * *} p<0.001$; Wilcoxon test.

$(13.9 \pm 3.6 \mathrm{~ms})$. However, the delay from this initial response to the object-induced suppression was similar for paratrigeminal, trigeminal, and thalamic neurons $(p=0.1895$; Kruskal-Wallis test) and happened at $19.5 \pm 9.8 \mathrm{~ms}$ from the initial whisking response.

The processing leading to response suppression cannot start before the occurrence of the first responses to contact at the brainstem. In our data, the delay from the onset of the response of the T cells' population in each nucleus to the object-induced suppression in that nucleus was $14.1 \pm 10.2 \mathrm{~ms}$ in the trigeminal nuclei, $14.8 \pm 5.3 \mathrm{~ms}$ in the $\mathrm{Pa} 5$, and $17.9 \pm 11.8 \mathrm{~ms}$ in the thalamus. Based on these data, the processing leading to suppression should last no more than $14.1 \mathrm{~ms}$ on average. If we consider the possibility that this processing was initiated by contact responses in nucleus principalis, whose activity was not recorded here, then we should add $\sim 1 \mathrm{~ms}$ to this assessment (Sosnik et al., 2001). A time window of $15 \mathrm{~ms}$ may allow a processing loop via the somatosensory cortex, if we consider the fastest responses recorded in the involved pathways (Derdikman et al., 2006; Furuta et al., 2010; Malmierca et al., 2014). We thus tested whether such a possibility is plausible. Cortical neurons across layers 2-5 of S1 exhibit strong latency adaptation; their onset latencies to artificial touch (same protocol as here) increase by $10-13.6 \mathrm{~ms}$ on average already by the third whisking cycle at $5 \mathrm{~Hz}$ (Derdikman et al., 2006, their Fig. 8, Table 3). We thus analyzed the latencies of suppression onset in our W-T neurons. As shown in Figure 8D (bottom), the suppression onset at the brainstem does not follow the latency adaptation profile measured at the cortex under the same conditions. Rather, it follows the typical latency dynamics of brainstem neurons (Fig. $8 D$, top), with the exception of a strong deviation of $\mathrm{Pa} 5$ latency in cycle 4 . In contrast to the behavior of cortical neurons (Derdikman et al., 2006, their Fig. 8, Table 3), no significant differences were found in the delay between contact onset and object-induced suppression between the first and last whisking cycles of the whisking train $(p=0.52$ for trigeminal cells and $p=0.81$ for paratrigeminal cells; Wilcoxon test). We thus conclude that the processing of response suppression does not depend on cortical responses, and thus is most likely completed locally at the brainstem, possibly involving subcortical loops of total delays $<15 \mathrm{~ms}$.

\section{Discussion}

In the current study, we compared the neuronal responses of cells located in the trigeminal and paratrigeminal nuclei to free-air whisking and whisking against an object, thus providing the first account of whisking and touch selectivity in the brainstem trigeminal complex and the first evidence of the encoding of vibrissal-related sensory information in the Pa5. In general, our results show that $\mathrm{W}$ responses are matured already at the brainstem level, $\mathrm{T}$ and $\mathrm{W}+\mathrm{T}$ responses are partially matured in the brainstem, and $\mathrm{W}-\mathrm{T}$ responses are likely generated at the brainstem. 

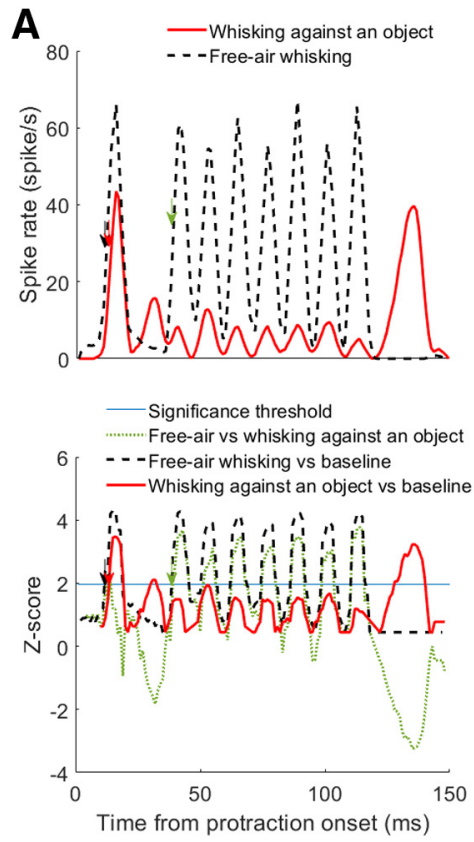
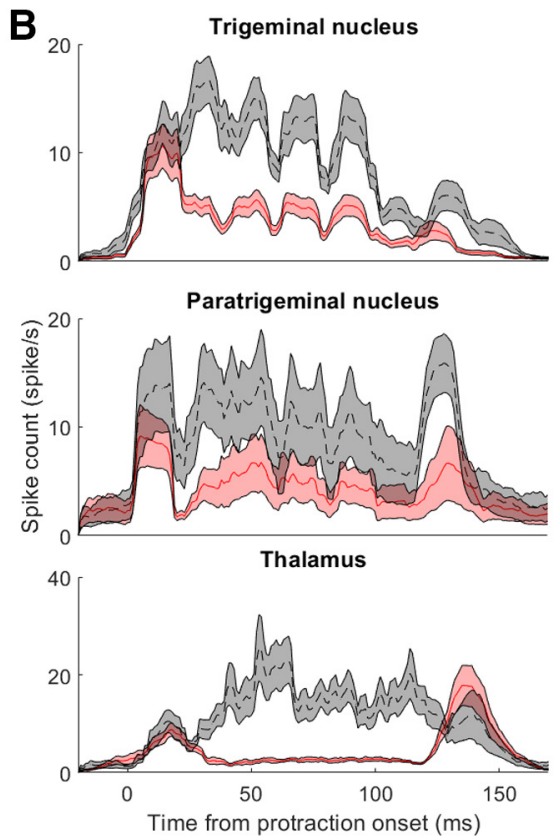
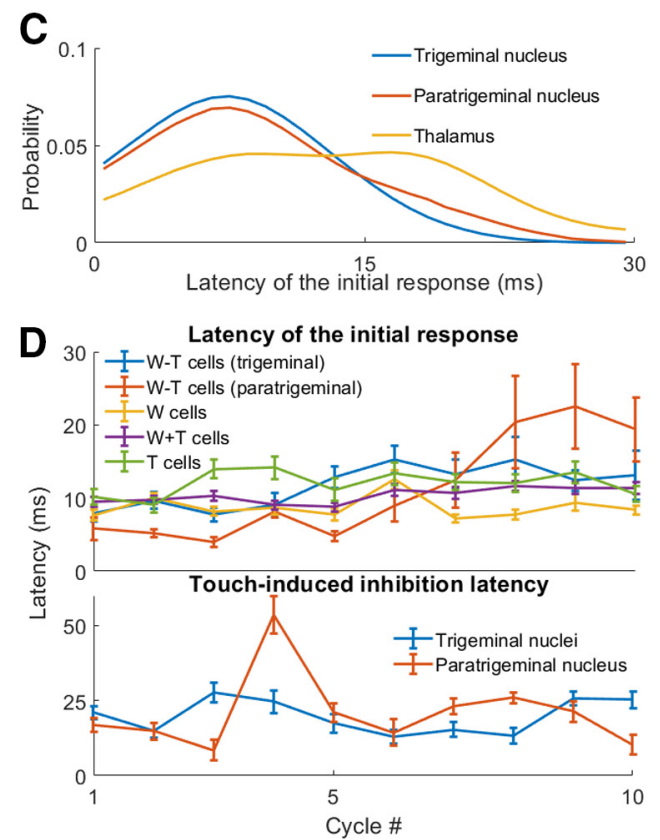

Figure 8. Object-induced suppression of the whisking response to whisker motion for W-T cells. $\boldsymbol{A}$, Computation of the times of the initial response and of the object-induced suppression for W-T cells. Top, The response dynamics to whisking in free-air and against an object for one of the recorded units. Bottom, Corresponding $z$ scores. The times where the $z$ scores crossed the significance threshold are reported on the top using corresponding colored arrows. B, Averaged PSTHs aligned to protraction onset for W-T cells recorded in the trigeminal nucleus (top), in the paratrigeminal nucleus (middle), and in the thalamus (bottom). Black -free air whisking, red -whisking against the object. Shaded areas represent the SEM. $\boldsymbol{C}$, Distribution of the latencies to the half-peak (in ms) from protraction onset for W-T cells recorded in the trigeminal nucleus (blue), in the paratrigeminal nucleus (red), or in the thalamus (orange). $\boldsymbol{D}$, Evolution of the average response (top) and inhibition (bottom) latencies during a train of 10 consecutive whisking cycles. The response latencies were measured from contact onset to the half-peak for $T$ cells and from protraction onset to the half-peak for the rest of the cells. The suppression latencies were computed as the delay between contact onset to the object-induced suppression (defined based on the $z$ score; see $\boldsymbol{A}$ ). Error bars indicate SEM.

Spatial selectivity is conserved in the extralemniscal pathway but not in the paralemniscal pathway

If only monowhisker cells are found in the TG (Szwed et al., 2003), this is not the case in the trigeminal and paratrigeminal nuclei, where many cells respond to more than one whisker. The size of the receptive field did not appear to vary greatly between the considered brainstem nuclei, although cells with very large receptive field, spanning up to three whisker rows, were found only in SpVor while SpVir seemed to have the higher spatial resolution. However, this last finding did not hold true at the thalamic level. This was because the average size of the receptive field increased dramatically between the brainstem and the thalamus in the paralemniscal pathway while it remained mostly unchanged in the extralemniscal pathway, resulting in receptive fields that were twice as large in POm compared with VPMvl (Fig. 6C). We note that this difference in spatial resolution between the two pathways can be easily explained from a functional point of view: since the extralemniscal pathway is hypothesized to carry information about contact with external objects, the identity of the contacting whisker is expected to be required to infer the location and shape of the object.

\section{The neuronal activity in SpVi is consistent with its thalamic projections}

Cells in SpVir encoded mostly proprioceptive information while SpVic neurons responded preferentially to touch (Fig. 6A). These findings are consistent with previous thalamic observations (C. Yu et al., 2006). The distribution of touch selectivity in SpVir was nearly identical to that observed in POm and significantly different from that observed in the TG (Fig. 6A). This result indicates that most of the sensory processing along the paralemniscal pathway occurs at the brainstem level in SpVir; this processing is likely primarily based on selection of $\mathrm{W}$ primary afferents. This was not the case in the extralemniscal pathway (from SpVic to VPMvl), where the proportion of touch units in the population at the thalamic level was $>3$ times greater than that observed at the brainstem level $(69 \%$ and $19 \%$, respectively; Fig. 6A). It hence appears that the selective encoding of active sensing along the trigeminal pathways becomes matured at different levels, possibly reflecting different phases of evolution. The similarity in response between the brainstem and thalamic stations of the paralemniscal pathway suggests that no further selectivity was added here on thalamic evolution. However, additional processing, or selection processes, occurs between SpVic and VPMvl. Such increased selectivity might be required for increased accuracy in the processing of external objects.

\section{The anatomic division of $\mathrm{SpVo}$ into a rostral and caudal part does not appear to be functional}

In a pioneering study in the rabbit, Meessen and Olszewski (1949) divided SpVo into its rostral and caudal parts. They characterized the caudal part of SpVo as "distinctly poorer in cells," where in addition to a few larger cells, many spindle-shaped and triangular elements and some smaller round cells were found. Falls (1984) and Jacquin and Rhoades (1990) also reported that the majority of neurons were larger in the rostral part of SpVo. Later, Shigenaga and Yoshida (2007) reported a division of SpVo along its rostro-caudal axis into three subnuclei in the cat and into two subnuclei in the rat. The neurons in the rostral part of SpVo were characterized as "large multipolar cells," whereas the caudal part of SpVo was composed of oval- or spindle-shaped 

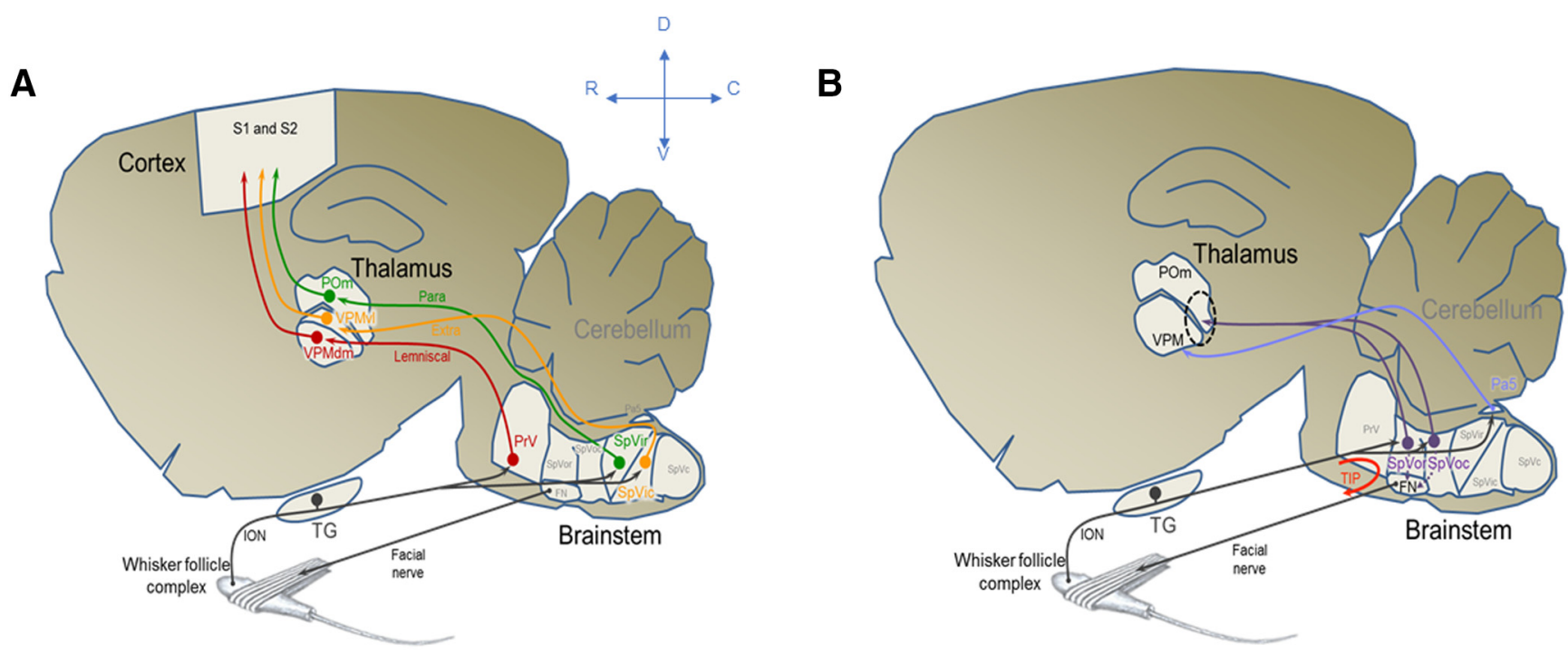

Figure 9. Vibrissal sensory afferent pathways, from the TG to the sensory cortex. A, Current state of knowledge. The extralemniscal pathway, carrying touch information from SpVic, is represented in orange. The paralemniscal pathway which starts in SpVir (green) encodes whisking information, whereas the lemniscal pathway (red) contains a combination of whisking and touch information. $\boldsymbol{B}$, New sensory pathways identified in the present study. SpVor and SpVoc (purple) carry touch information to the most caudal parts of POm and VPM. Pa5 (blue) encodes a complex combination of touch and whisking information and projects to the VPM (to which part of the nucleus is yet to be determined). We also proposed that an MSM (motor-sensory-motor) loop, the TIP loop (red), is mediated by either SpVor or SpVoc. ION, infra-orbital nerve; FN, facial nucleus.

small cells, triangular or fusiform medium-sized cells, and sparsely scattered large multipolar cells. However, it is not known whether morphologic heterogeneity of the SpVo subdivisions brings to bear influence on their specific function. Studying processing of the whisking and touch signals in the rat SpVo, we did not observe significant differences in the size of the neuronal receptive field or in the active sensing selectivity between the rostral and caudal parts of SpVo that were reported to contain neurons of different size.

\section{SpVo contains a high proportion of cells responding preferentially to touch}

Both SpVor and SpVoc contained the highest proportion of $\mathrm{T}$ cells across the nuclei we considered (Table 1). SpVo does not project to VPMvl, which is the touch-encoding thalamic extralemniscal region (C. Yu et al., 2006), but to the most caudal regions of the VPM and POm (Jacquin and Rhoades, 1990; Veinante et al., 2000) for which electrophysiological data are not yet available (Deschênes and Urbain, 2009). Our findings thus suggest that this additional pathway encodes similar information to that encoded along the extralemniscal pathway.

The fact that SpVo encodes mostly touch information is in accordance with Bellavance et al. (2017) who proposed that SpVo and SpVir may be both involved in touch-induced pump (TIP) generation, a fast, reflexive modulation of the whiskers motion happening on contact with an object (Deutsch et al., 2012; Sherman et al., 2017). Their model stipulates that SpVo is responsible for a transient inhibition followed by an activation of the intrinsic protracting muscles while SpVir activates the extrinsic retracting muscles. Our study shows that SpVir encodes only proprioceptive information and hence could not be responsible for a TIP reflex. Since SpVo, both in its rostral and caudal parts, encodes preferentially touch information, we propose that it is the main drive for TIPs generation.

\section{Pa5 encodes vibrissal sensory information}

Advancing microelectrodes through Pa5, we found 33 cells in the course of seven experiments which responded to free-air whisking and/or whisking against an object. Nearly one-third (30.3\%) of the cells responded exclusively to a single whisker. This nucleus presented a very wide distribution of touch selectivity, with cells covering the whole range of TIs, indicating a significant involvement in the processing of active vibrissal touch. In particular, the intriguing $\mathrm{W}$-T coding, whose role in tactile perception is not yet clear, is prominent in $\mathrm{Pa} 5$ (Fig. 6A).

Because of its previously known integration of respiratory and cardiovascular information (Couture and Lindsey, 2000; Buck et al., 2001; Y. G. Yu et al., 2002; Junior et al., 2004; Caous et al., 2008; Sousa and Lindsey, 2009), we propose that Pa5 could be involved in the coordination of respiratory and whisking rhythms through its projections to the reticular formation, the ambiguus nucleus and the parabrachial nucleus. Additional studies are needed to determine to which part of the thalamic VPM nucleus $\mathrm{Pa} 5$ projects. Meanwhile, it remains unclear whether $\mathrm{Pa} 5$ is part of the lemniscal (as the abundance of W-T cells suggests), extralemniscal or of an unknown pathway. We hope these results will pave the way to new studies investigating the role of $\mathrm{Pa} 5$ in vibrissal perception.

\section{Neuronal adaptation to the whisking and touch stimuli increases the cell response selectivity}

On average, the recorded cells decreased their activity in response to consecutive whisking cycles. The magnitude of this decline depended on the cell type and on the presence of an object in the whisking field. The response adaptation led to significantly higher TIs in the last whisking stimulation cycles for $\mathrm{T}$ and $\mathrm{W}+\mathrm{T}$ cells and to even more negative TIs for $\mathrm{W}-\mathrm{T}$ cells (Fig. 7). Thus, adaptation increased the neuronal selectivity to the preferred stimulus. Such increased selectivity may facilitate perceptual processes during the sequences of object palpations observed in behaving rats (Carvell and Simons, 1990; Berg and Kleinfeld, 2003; Knutsen et al., 2006; Mitchinson et al., 2007; Saraf-Sinik et al., 2015).

The adaptive mechanism that increases selectivity may reflect a general rule proposed for increasing sensory signal-to-noise ratios in afferent processing by Mohar et al. (2013, 2015). By 
studying adaptation in interpolaris and principalis neurons, these authors demonstrated that a mechanism that originates at the synaptic level induces stronger adaptation for nonpreferred stimuli and weaker for preferred ones.

\section{$\mathrm{W}$ - $\mathrm{T}$ responses originate in the brainstem}

In the course of this experiment, $18 \mathrm{~W}$-T cells were recorded, for which the neuronal activity was suppressed in the presence of an object. W-T cells are not found in the TG (Szwed et al., 2003) and must therefore result from computations downstream. These cells were mostly found in the Pa5 and SpVi, with occasional occurrences in SpVo. The proportion of $\mathrm{W}$-T cells in $\mathrm{Pa} 5$ was even higher than previously observed in VPMdm (C. Yu et al., 2006).

As we showed in delay of the object-induced suppression in $\mathrm{W}$-T cells, the suppression onsets at the thalamus lagged those in the brainstem by 3-4 ms on average. This lagging precludes the possibility that brainstem suppression is indirectly inherited from the thalamus. In contrast, it suggests that thalamic suppression is inherited from the brainstem. Can brainstem suppression be generated by some neuronal loop via the cortex? We showed that the processing leading to the suppression of the response of brainstem W-T cells should be completed within $15 \mathrm{~ms}$, on average. This time window does not preclude a cortical inhibition mechanism (Derdikman et al., 2006; Furuta et al., 2010; Malmierca et al., 2014). However, Derdikman et al. (2006) have shown that $\mathrm{S} 1$ response latencies increased in consecutive whisking cycles. The fact that the object-induced suppression observed in our study did not follow a similar trend (Fig. 8D) is a strong indication that the generation of response suppression in brainstem $\mathrm{W}$-T cells does not involve cortical processing. This indication, and the fact that intra-brainstem inhibition is a key processing factor in the vibrissal system (Furuta et al., 2008), suggest that also active-touch induced inhibition (W-T) is generated within brainstem circuits.

\section{Comparison with results from awake behaving rats}

We have chosen to perform this study using artificial whisking in anesthetized animals. This method offers the advantage of highly controlled, repeatable stimulation that allows for the systematic comparison between regions described here. However, several key differences between this method and awake whisking should be acknowledged. First, artificial whisking is generated by synchronous activation of only the whisking pad's intrinsic muscle group, whereas natural whisking patterns involve several extrinsic groups as well (Berg and Kleinfeld, 2003). Second, as the motion is generated peripherally, motor-related corollary discharge activity that may play a role in sensory processing is absent in artificial whisking. Last, urethane anesthesia used in this method may also alter sensory processing. Therefore, the studied neuronal populations may display different characteristics in awake behavior, especially when different contexts are considered (Isett and Feldman, 2020). However, it should be noted that several key findings obtained using this method (C. Yu et al., 2006; Wallach et al., 2016) were later validated in awake animals (Moore et al., 2015; Severson et al., 2017), demonstrating the ability of this method to expose the information conveyed along the vibrissal afferent pathways. The results presented in this contribution are indicative of the ascending sensory drive propagating through the various pathways and its processing. While this ascending activity may drive several feedback pathways projecting back to the brainstem and influencing its activity, anesthesia abolishes any descending corollary discharge from motor or cognitive central regions projecting to the brainstem during awake behavior (Dauvergne et al., 2004; Furuta et al., 2010; Malmierca et al., 2014; Smith et al., 2015). Therefore, comparison of these results with those obtained in awake behaving rats could provide insight to the role of these top-down modulations. For instance, while we found the paralemniscal pathway to be predominantly driven by whisking afferents (Fig. 6A), encoding of self-motion in the paralemniscal thalamus was relatively poor in awake, head-fixed rats (Moore et al., 2015; Urbain et al., 2015). Future studies could explore the possibility that, as shown for other modalities (Roberts and Bell, 2000), descending input or feedback either gates or cancels this activity, perhaps in a context-dependent manner (Wallach et al., 2020).

In conclusion, this first comparative study of whisking and touch selectivity in the brainstem's trigeminal complex both confirms previous conjectures and provides new insights. We have confirmed that SpVir and SpVic encode whisking and touch information, respectively, as was predicted from their thalamic projections (Fig. 9A). Our study also shed light on two additional pathways, one originating from $\mathrm{SpVo}$ and the other from $\mathrm{Pa} 5$ (Fig. 9B). It provided the first indication that the SpVo encodes touch information and thus confirmed its previously hypothesized role in a touch reflexive loop (the TIP loop). Our work also brought to light, for the first time, the involvement of $\mathrm{Pa} 5$ in vibrissal information processing and demonstrated different maturation profiles for whisking and touch afferent encoding. Finally, our results indicate that the processing leading to touchtriggered suppression are implemented subcortically, likely within the brainstem.

\section{References}

Bellavance MA, Takatoh J, Lu J, Demers M, Kleinfeld D, Wang F, Deschênes M (2017) Parallel inhibitory and excitatory trigemino-facial feedback circuitry for reflexive vibrissa movement. Neuron 95:673-682.e4.

Berg RW, Kleinfeld D (2003) Rhythmic whisking by rat: retraction as well as protraction of the vibrissae is under active muscular control. J Neurophysiol 89:104-117.

Bon K, Lantéri-Minet M, Menétrey D (1997) Involvement of the dorsal paratrigeminal nucleus in pain-related phenomena. C R Acad Sci III 320:533539.

Boubenec Y, Shulz DE, Debregeas G (2012) Whisker encoding of mechanical events during active tactile exploration. Front Behav Neurosci 6:74.

Buck HS, Caous CA, Lindsey CJ (2001) Projections of the paratrigeminal nucleus to the ambiguus, rostroventrolateral and lateral reticular nuclei, and the solitary tract. Auton Neurosci 87:187-200.

Caous CA, Buck HS, Lindsey CJ (2001) Neuronal connections of the paratrigeminal nucleus: a topographic analysis of neurons projecting to bulbar, pontine and thalamic nuclei related to cardiovascular, respiratory and sensory functions. Auton Neurosci 94:14-24.

Caous CA, Koepp J, Couture R, Balan AC, Lindsey CJ (2008) The role of the paratrigeminal nucleus in the pressor response to sciatic nerve stimulation in the rat. Auton Neurosci 140:72-79.

Carvell GE, Simons DJ (1990) Biometric analyses of vibrissal tactile discrimination in the rat. J Neurosci 10:2638-2648.

Castro-Alamancos MA (2002) Different temporal processing of sensory inputs in the rat thalamus during quiescent and information processing states in vivo. J Physiol 539:567-578.

Chan-Palay V (1978) The paratrigeminal nucleus: I. Neurons and synaptic organization. J Neurocytol 7:405-418.

Couture R, Lindsey CJ (2000) Brain kallikrein-kinin system: from receptors to neuronal pathways and physiological functions. Handb Chem Neuroanat 16:241-300.

Dauvergne C, Ndiaye A, Buisseret-Delmas C, Buisseret P, Vanderwerf F, Pinganaud G (2004) Projections from the superior colliculus to the trigeminal system and facial nucleus in the rat. J Comp Neurol 478:233247. 
Derdikman D, Yu C, Haidarliu S, Bagdasarian K, Arieli A, Ahissar E (2006) Layer-specific touch-dependent facilitation and depression in the somatosensory cortex during active whisking. J Neurosci 26:9538-9547.

Deschênes M, Urbain N (2009) Vibrissal afferents from trigeminus to cortices. Scholarpedia 4:7454.

Deschênes M, Timofeeva E, Lavallee P (2003) The relay of high-frequency sensory signals in the whisker-to-barreloid pathway. J Neurosci 23:67786787.

Deutsch D, Pietr M, Knutsen PM, Ahissar E, Schneidman E (2012) Fast feedback in active sensing: touch-induced changes to whisker-object interaction. PLoS One 7:ee42272.

Diamond ME, Von Heimendahl M, Knutsen PM, Kleinfeld D, Ahissar E (2008) "Where" and "what" in the whisker sensorimotor system. Nat Rev Neurosci 9:601-612.

Ebara S, Kumamoto K, Matsuura T, Mazurkiewicz JE, Rice FL (2002) Similarities and differences in the innervation of mystacial vibrissal follicle-sinus complexes in the rat and cat. J Comp Neurol 449:103119.

Falls WM (1984) The morphology of neurons in trigeminal nucleus oralis projecting to the medullary dorsal horn (trigeminal nucleus caudalis): retrograde horseradish peroxidase and Golgi study. Neuroscience 13:12791298.

Furuta T, Nakamura K, Deschênes M (2006) Angular tuning bias of vibrissaresponsive cells in the paralemniscal pathway. J Neurosci 26:1054810557.

Furuta T, Timofeeva E, Nakamura K, Okamoto-Furuta K, Togo M, Kaneko T, Deschênes M (2008) Inhibitory gating of vibrissal inputs in the brainstem. J Neurosci 28:1789-1797.

Furuta T, Urbain N, Kaneko T, Deschênes M (2010) Corticofugal control of vibrissa-sensitive neurons in the interpolaris nucleus of the trigeminal complex. J Neurosci 30:1832-1838.

Gibson JM (1987) A quantitative comparison of stimulus-response relationships of vibrissa-activated neurons in subnuclei oralis and interpolaris of the rat's trigeminal sensory complex: receptive field properties and threshold distributions. Somatosens Res 5:135-155

Haidarliu S, Ahissar E (2001) Size gradients of barreloids in the rat thalamus. J Comp Neurol 429:372-387.

Haidarliu S, Simony E, Golomb D, Ahissar E (2011) Collagenous skeleton of the rat mystacial pad. Anat Rec (Hoboken) 294:764-773.

Hartigan PM (1985) Algorithm AS 217: computation of the dip statistic to test for unimodality. Appl Stat 34:320-325.

Hartigan JA, Hartigan PM (1985) The dip test of unimodality. Ann Stat 13:70-84.

Henderson TA, Jacquin MF (1995) What makes subcortical barrels? In: The barrel cortex of rodents, Springer, Boston, MA. pp 123-187.

Isett BR, Feldman DE (2020) Cortical coding of whisking phase during surface whisking. Curr Biol 30:3065-3074.

Jacquin MF, Rhoades RW (1990) Cell structure and response properties in the trigeminal subnucleus oralis. Somatosens Mot Res 7:265-288.

Jacquin MF, Woerner D, Szczepanik AM, Riecker V, Mooney RD, Rhoades RW (1986) Structure-function relationships in rat brainstem subnucleus interpolaris: I. Vibrissa primary afferents. J Comp Neurol 243:266-279.

Junior AB, Caous CA, Yu YG, Lindsey CJ (2004) Barosensitive neurons in the rat tractus solitarius and paratrigeminal nucleus: a new model for medullary, cardiovascular reflex regulation. Can J Physiol Pharmacol 82:474-484.

Kleinfeld D, Deschênes M (2011) Neuronal basis for object location in the vibrissa scanning sensorimotor system. Neuron 72:455-468.

Knutsen PM, Pietr M, Ahissar E (2006) Haptic object localization in the vibrissal system: Behavior and Performance. J Neurosci 26:8451-8464.

Koepp J, Lindsey CJ, Motta EM, Rae GA (2006) Role of the paratrigeminal nucleus in nocifensive responses of rats to chemical, thermal and mechanical stimuli applied to the hind paw. Pain 122:235-244.

Ma WL, Zhang WB, Feng G, Cai YL (2005) Calbindin D28k-containing neurons in the paratrigeminal nucleus receive convergent nociceptive information and project to nucleus of the solitary tract in rat. Brain Res 1038:132-140.

Malmierca E, Chaves-Coira I, Rodrigo-Angulo M, Nuñez A (2014) Corticofugal projections induce long-lasting effects on somatosensory responses in the trigeminal complex of the rat. Front Syst Neurosci 8:100.
Marfurt CF, Rajchert DM (1991) Trigeminal primary afferent projections to "non-trigeminal" areas of the rat central nervous system. J Comp Neurol 303:489-511.

Meessen H, Olszewski J (1949) A cytoarchitectonic atlas of the rhombencephalon of the rabbit. New York: Karger.

Mitchinson B, Martin CJ, Grant RA, Prescott TJ (2007) Feedback control in active sensing: rat exploratory whisking is modulated by environmental contact. Proceedings of the Royal Society B: Biological Sciences 274:1035-1041.

Mohar B, Katz Y, Lampl I (2013) Opposite adaptative processing of stimulus intensity in two major nuclei of the somatosensory brainstem. J Neurosci 33:15394-15400.

Mohar B, Ganmor E, Lampl I (2015) Faithful representation of tactile intensity under different contexts emerges from the distinct adaptive properties of the somatosensory relay stations. J Neurosci 35:69977002 .

Moore JD, Lindsay NM, Deschênes M, Kleinfeld D (2015) Vibrissa selfmotion and touch are reliably encoded along the same somatosensory pathway from brainstem through thalamus. PLoS Biol 13: e1002253.

Moreno A, Garcia-Gonzalez V, Sanchez-Jimenez A, Panetsos F (2005) Principalis, oralis and interpolaris responses to whisker movements provoked by air jets in rats. NeuroReport 16:1569-1573.

Paxinos G, Watson C (2006) The rat brain in stereotaxic coordinates, Ed. 6. San Diego: Academic.

Pierret T, Lavallee P, Deschênes M (2000) Parallel streams for the relay of vibrissal information through thalamic barreloids. J Neurosci 20:74557462 .

Quist BW, Seghete V, Huet LA, Murphey TD, Hartmann MJ (2014) Modeling forces and moments at the base of a rat vibrissa during noncontact whisking and whisking against an object. J Neurosci 34:98289844.

Roberts PD, Bell CC (2000) Computational consequences of temporally asymmetric learning rules: II. Sensory image cancellation. J Comput Neurosci 9:67-83.

Saraf-Sinik I, Assa E, Ahissar E (2015) Sensory strategy underlies the perception of object location in rats. J Neurosci 35:8777-8789.

Saxon DW, Hopkins DA (1998) Efferent and collateral organization of paratrigeminal nucleus projections: an anterograde and retrograde fluorescent tracer study in the rat. J Comp Neurol 402:93-110.

Severson KS, Xu D, Van de Loo M, Bai L, Ginty DD, O'Connor DH (2017) Active touch and self-motion encoding by Merkel cell-associated afferents. Neuron 94:666-676.e9.

Sherman D, Oram T, Harel D, Ahissar E (2017) Attention robustly gates a closed-loop touch reflex. Curr Biol 27:1836-1843.e7.

Shigenaga Y, Yoshida A (2007) Trigeminal brainstem nuclear complex, anatomy. In: Encyclopedia of pain (Schmidt R, Willis W, eds). Berlin: Springer.

Simony E, Bagdasarian K, Herfst L, Brecht M, Ahissar E, Golomb D (2010) Temporal and spatial characteristics of vibrissa responses to motor commands. J Neurosci 30:8935-8952.

Smith JB, Watson GD, Alloway KD, Schwarz C, Chakrabarti S (2015) Corticofugal projection patterns of whisker sensorimotor cortex to the sensory trigeminal nuclei. Front Neural Circuits 9:53.

Sosnik R, Haidarliu S, Ahissar E (2001) Temporal frequency of whisker movement: I. Representations in brain stem and thalamus. J Neurophysiol 86:339-353.

Sousa LO, Lindsey CJ (2009) Cardiovascular and baroreceptor functions of the paratrigeminal nucleus for pressor effects in non-anaesthetized rats. Auton Neurosci 147:27-32.

Sugimoto T, Fujiyoshi Y, He YF, Xia C, Ichikawa H (1997) Trigeminal primary projection to the rat brain stem sensory trigeminal nuclear complex and surrounding structures revealed by anterograde transport of cholera toxin B subunit-conjugated and Bandeiraea simplicifolia isolectin B4-conjugated horseradish peroxidase. Neurosci Res 28:361-371.

Szwed M, Bagdasarian K, Ahissar E (2003) Encoding of vibrissal active touch. Neuron 40:621-630.

Tonomura S, Ebara S, Bagdasarian K, Uta D, Ahissar E, Meir I, Lampl I, Kuroda D, Furuta T, Furue H, Kumamoto K (2015) Structure-function correlations of rat trigeminal primary neurons: emphasis on club-like 
endings, a vibrissal mechanoreceptor. Proc Jpn Acad Ser B Phys Biol Sci 91:560-576.

Urbain N, Salin PA, Libourel PA, Comte JC, Gentet LJ, Petersen CC (2015) Whisking-related changes in neuronal firing and membrane potential dynamics in the somatosensory thalamus of awake mice. Cell Rep 13:647-656.

Veinante P, Jacquin MF, Deschênes M (2000) Thalamic projections from the whisker-sensitive regions of the spinal trigeminal complex in the rat. J Comp Neurol 420:233-243.

Wallach A, Bagdasarian K, Ahissar E (2016) On-going computation of whisking phase by mechanoreceptors. Nat Neurosci 19:487-493.

Wallach A, Deutsch D, Oram T, Ahissar E (2020) Predictive whisker kinematics reveal context-dependent sensorimotor strategies. PLoS Biol 18:e3000571.
Williams MN, Zahm DS, Jacquin MF (1994) Differential foci and synaptic organization of the principal and spinal trigeminal projections to the thalamus in the rat. Eur J Neurosci 6:429-453.

Yu C, Derdikman D, Haidarliu S, Ahissar E (2006) Parallel thalamic pathways for whisking and touch signals in the rat. PLoS Biol 4: e124.

Yu C, Horev G, Rubin N, Derdikman D, Haidarliu S, Ahissar E (2015) Coding of object location in the vibrissal thalamocortical system. Cereb Cortex 25:563-577.

Yu YG, Caous CA, Balan AC, Rae GA, Lindsey CJ (2002) Cardiovascular responses to sciatic nerve stimulation are blocked by paratrigeminal nucleus lesion. Auton Neurosci 98:70-74. 